HETEROCYCLES, Vol. 97, No. 2, 2018, pp. 729 - 743. @ 2018 The Japan Institute of Heterocyclic Chemistry Received, 10th February, 2018, Accepted, 28th February, 2018, Published online, 5th March, 2018 DOI: $10.3987 / \mathrm{COM}-18-\mathrm{S}(\mathrm{T}) 42$

\title{
ß-SELECTIVE D-PSICOFURANOSYLATION OF PYRIMIDINE BASES AND THIOLS
}

\section{Atsushi Ueda, ${ }^{1,2} *$ Yuri Nishimura, ${ }^{1}$ Yui Makura, ${ }^{2}$ Masakazu Tanaka, ${ }^{2}$ and Jun'ichi Uenishi ${ }^{1,3}$}

${ }^{1}$ Kyoto Pharmaceutical University, Misasagi, Yamashina, Kyoto 607-8412, Japan

2 Graduate School of Biomedical Sciences, Nagasaki University, 1-14 Bunkyo-machi, Nagasaki 852-8521, Japan. E-mail: aueda@nagasaki-u.ac.jp (A. Ueda) ${ }^{3}$ Graduate School of Pharmaceutical Sciences, Osaka University, Suita, Osaka 565-0871, Japan.

\begin{abstract}
N$-Glycosidation of D-psicofuranosyl donor 1 with pyrimidine bases took place $\beta$-selectively in a $\beta / \alpha$-ratio of $8: 1 \sim 7: 1$. For $S$-glycosidation, 3,4-O-(3-pentylidene)-protected D-psicofuranosyl donor 15 was effective to increase $\beta$-selectivity up to $7: 1$.
\end{abstract}

\section{INTRODUCTION}

Rare sugars including L-glucose, L-ribose, D-allose, D-tagatose, and so on, attract many scientists by their unique properties. ${ }^{1} \mathrm{D}$-Psicose is one of the D-hexuloses and is also categorized into rare sugar due to its rare occurrence in nature. D-Psicose and its analogs have shown a variety of biological activities regarding anti-oxidant, ${ }^{2}$ inhibitors against $\alpha$-glucosidase ${ }^{3,4}$ and $N$-acetylglycosyltransferase, ${ }^{5}$ and anti-tumor activities. ${ }^{6}$ However, limited supply and expensive price restrict development of their use and research. In 1993, Izumori et al. have found the specific isomerase for the transformation of D-fructose to D-psicose. ${ }^{7}$ After developing this finding, recent successful mass-production has provided large quantities of D-psicose commercially available. ${ }^{8}$ Indeed, due to a specific property as a low-calorie sweetener, D-psicose attracts dietary patients ${ }^{9 a}$ and healthy consumers in food market to date. ${ }^{9 b}$ We have investigated $O$-glycosidation reactions of D-psicose $\mathrm{e}^{10,11}$ in which we reported the chemical synthesis of $\mathbf{1}$ from D-ribose, ${ }^{10}$ and found an excellent $\beta$-selectivity of $\mathbf{1}$ as an efficient D-psicofuranosyl donor with wide-range of glycosyl acceptors, such as monosaccharide, aliphatic alcohol, phenol, ceramide, and so forth (Scheme 1). ${ }^{11,12}$ In an extension of these studies, we report $\beta$-selective D-psicofuranosidation with pyrimidines and thiols, herein. 


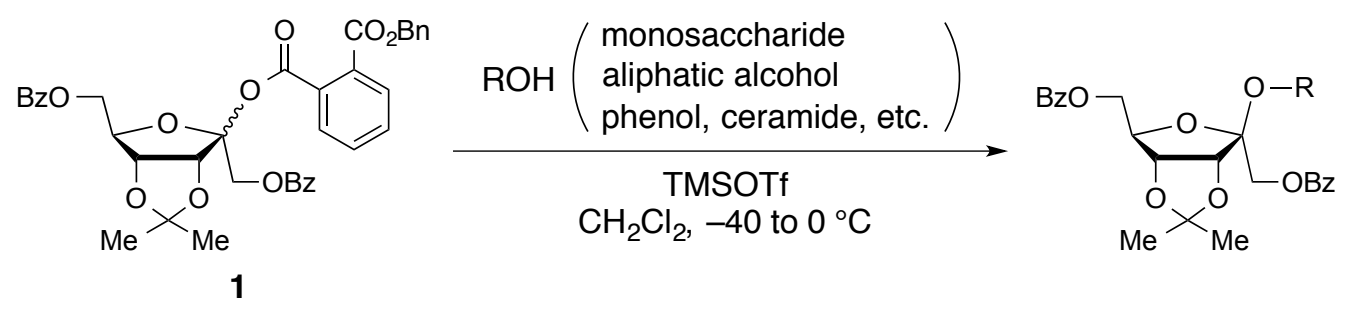

Scheme 1. $\beta$-Selective $O$-glycosylation of D-psicofuranose

\section{RESULTS AND DISCUSSION}

Preparation of 1 has been improved two step-shorter than the previous route ${ }^{10}$ (Scheme 2). D-Ribose derivative $2^{10}$ was first dihydroxylated to a triol $\mathbf{3}$, and one of the two secondary alcohols was oxidized selectively with the other intact under the conditions reported by Grindley et al. ${ }^{13}$ Then, selective mono-benzoylation of the resultant D-psicose 4 gave 5 with a 3:1 ratio of anomeric isomers.

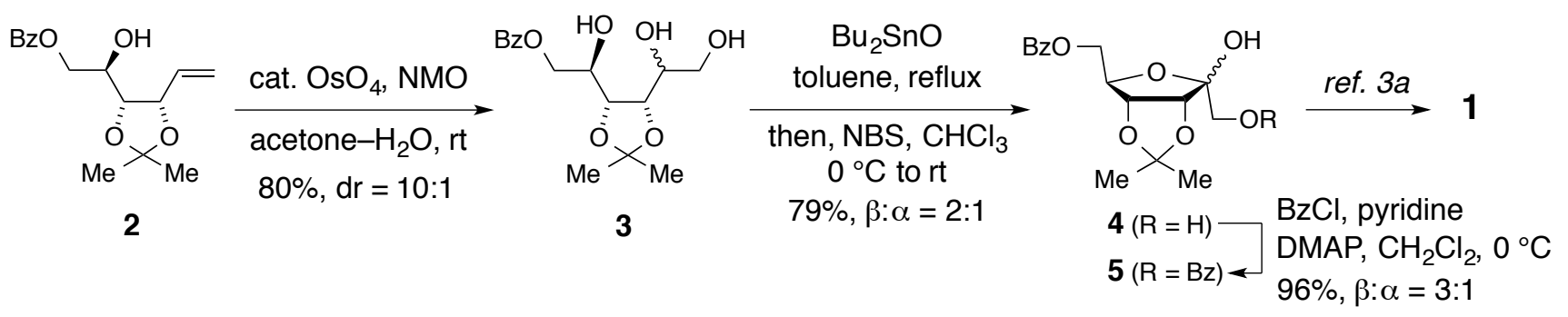

Scheme 2. Synthesis of glycosyl donor 1

We first examined $N$-glycosylation of uracil with 1 by Vorbrüggen method $^{14}$ (Table 1, entry 1). $O, O^{\prime}$-Bis(trimethylsilyl)uracil, generated in situ from uracil and $N, O$-bis-(trimethylsilyl)acetamide, was reacted with 1 using TMSOTf as a promoter to give 1-(D-psicofuranosyl)uracil 6 in $68 \%$ yield in a $\beta / \alpha$-ratio of 7:1. $N$-Psicofuranosylation of thymine, and $N^{4}$-benzoylcytosine also gave $N$-glycosides 7 and 8 in $91 \%$ and $72 \%$ yields with a $\beta / \alpha$-ratio of $8: 1$ and $7: 1$, respectively (entries 2 and 3 ). ${ }^{15}$ On the other hand, poor selectivities $(\beta / \alpha=3: 1 \sim 2: 1)$ were observed when thiophenol and 1-dodecanethiol were used as a glycosyl acceptor in $S$-psicofuranosidation (entries 4 and 5). This unsatisfied $\beta$-selectivity with thiol would arise from a size of glycosyl acceptor in primary thiol vs pyrimidine nucleobase. ${ }^{16}$ 
Table 1. Glycosylation of pyrimidines and thiols with D-psicofuranosyl donor $\mathbf{1}$
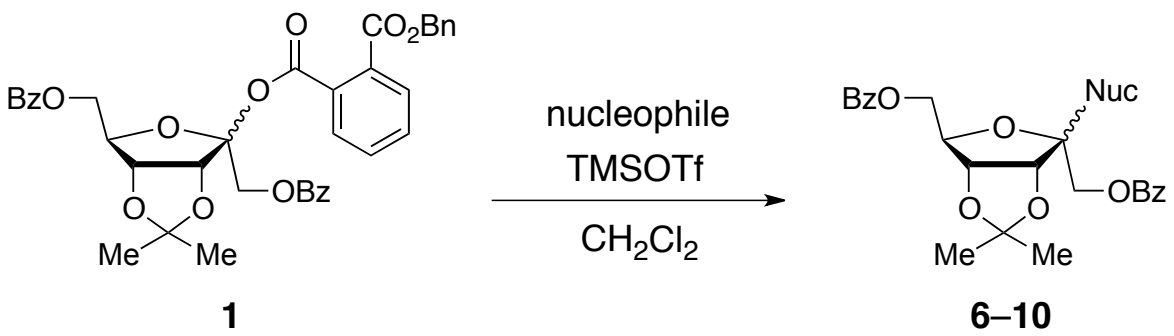

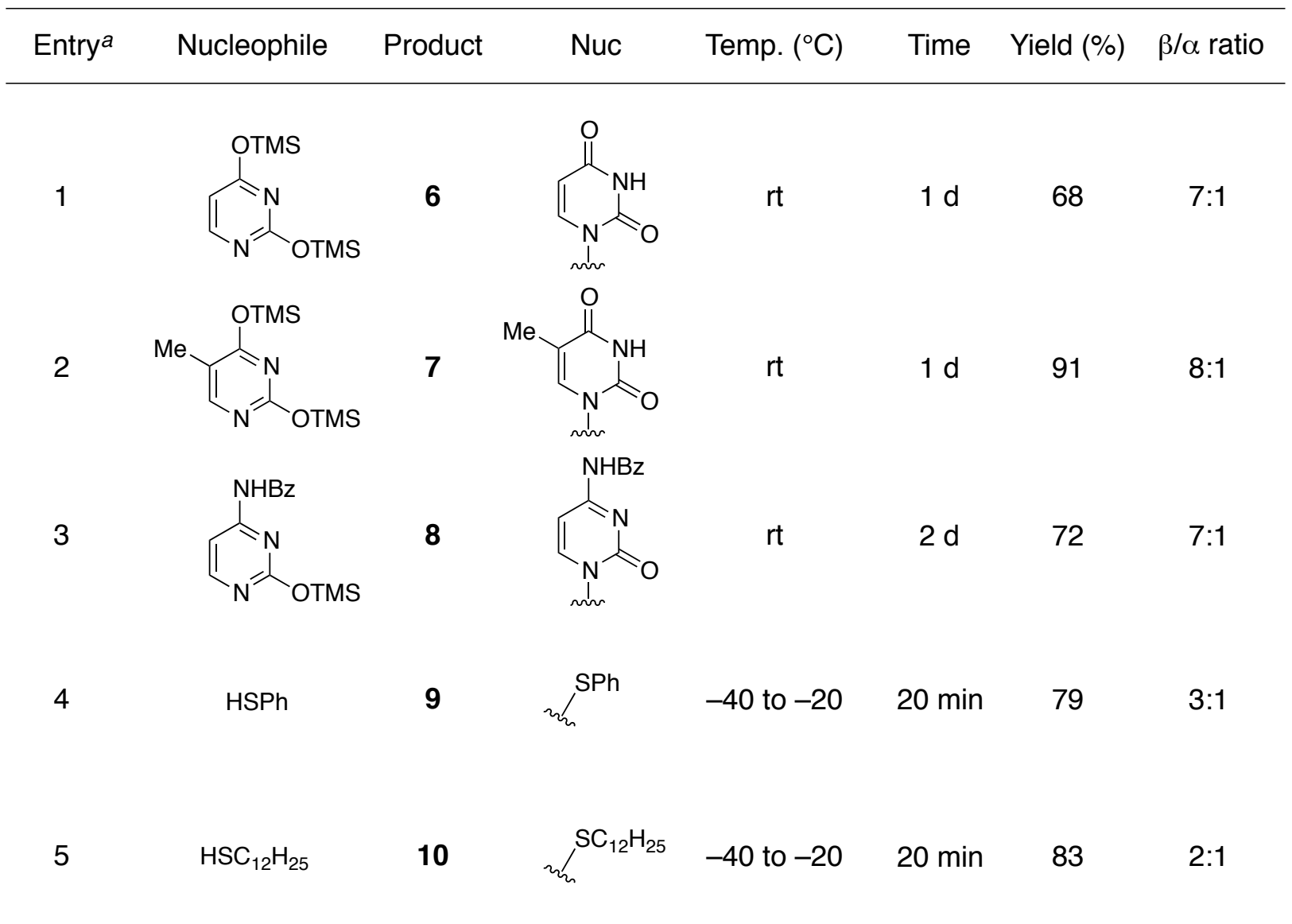

a Nucleophile (1.5 equiv) and TMSOTf (1.0 equiv) were used in $\mathrm{CH}_{2} \mathrm{Cl}_{2}(0.1 \mathrm{M})$.

The protecting group of 3,4-diol in $\mathbf{1}$ could play an important role for the facile formation of $\beta$-D-psicofuranoside. The acetonide group may prevent an access of the glycosyl acceptor from the $\alpha$-side. ${ }^{10,11}$ Based on this consideration, we used 3-pentylidene group in $\mathbf{1 5}$ as a larger glycosyl donor instead of acetonide. The synthesis from D-psicose is described in Scheme 3. First, primary alcohols of D-psicose were protected with TBDPSCl to give $\mathbf{1 1}$ in 93\% yield. Then, protection of C-3/4-diol as 3 -pentylidene group, ${ }^{17}$ followed by the replacement of two $O$-TBDPS groups with $O$-benzoyl groups by desilylation and benzoylation processes gave psicofuranose 14 in 69\% overall yield from 11 in three steps. Esterification of $\mathbf{1 4}$ with benzyl hydrogen phthalate by DCC provided $\mathbf{1 5}$ in $92 \%$ yield. $^{18}$ 

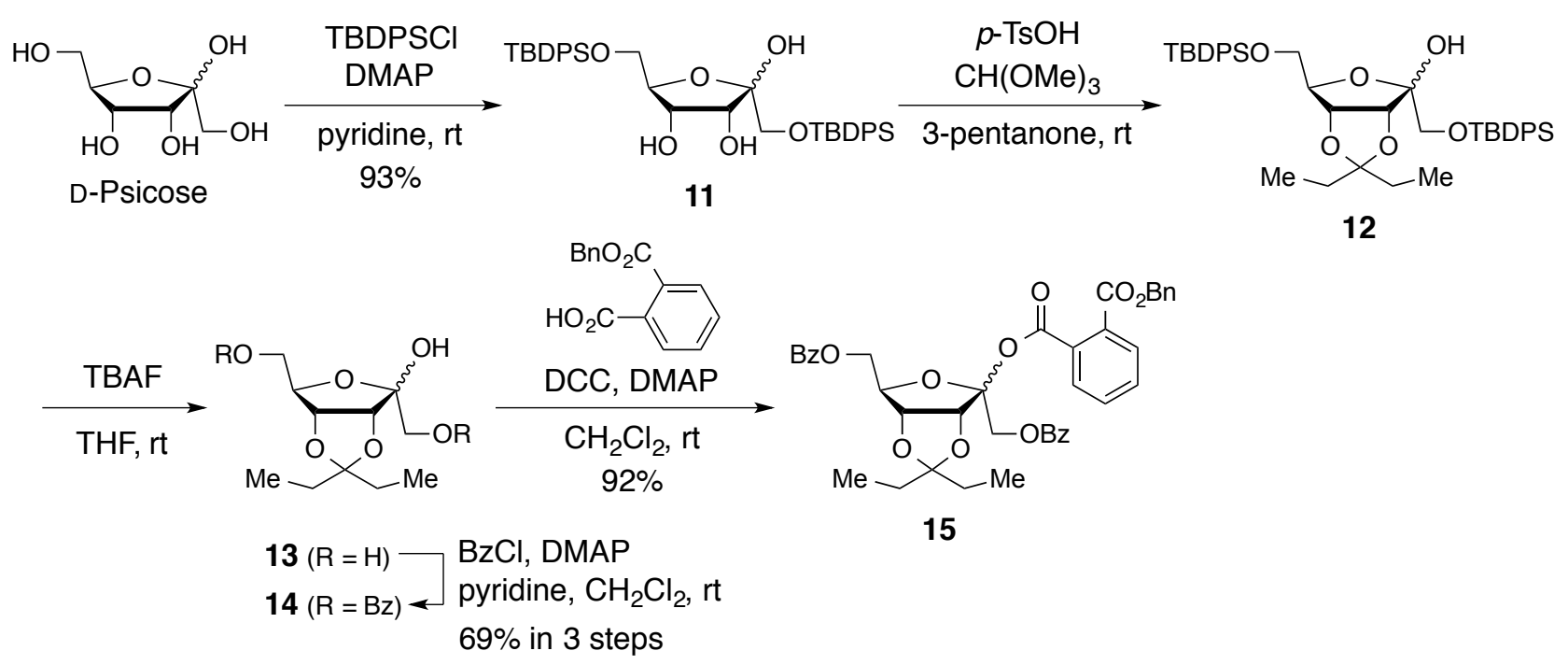

12

Scheme 3. Synthesis of 3,4-O-(3-pentylidene)-protected glycosyl donor 15

The glycosyl donor 15 was subjected to glycosidation with thiophenol. The thioglycoside $\mathbf{1 6}$ was obtained in $83 \%$ yield (Scheme 4). ${ }^{19}$ The selectivity was improved to be $7: 1$ from 3:1 ( $\beta / \alpha$-ratio). $\beta$-Selectivity of the reaction with 1-dodecanethiol was also increased to 7:1 from 2:1. On the other hand, glycosidation employing 15 for uracil, thymine, and $N^{4}$-benzoylcytosine resulted in the similar $\beta$-selectivities described in Table 1 with $\mathbf{1}$, albeit rather slower reaction rate. ${ }^{20}$

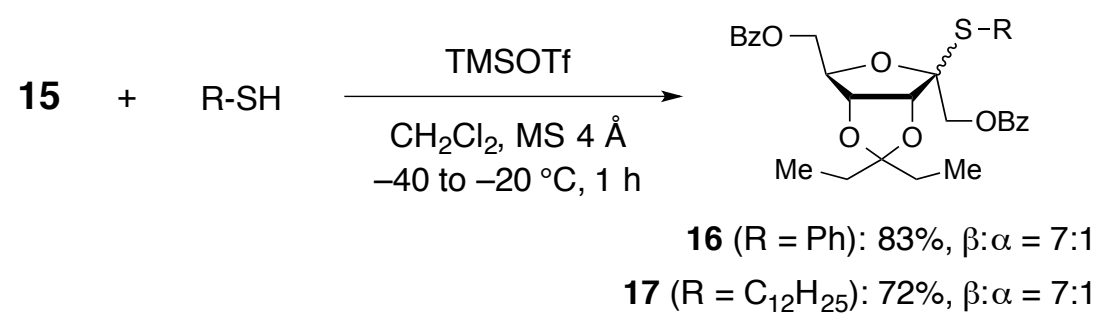

Scheme 4. S-Glycosidation of D-psicofuranosyl donor 15

Deprotection of $\beta$-D-psicofuranoside was shown in Scheme 5. Treatment of uridine derivative 6 with aqueous trifluoroacetic acid (TFA) gave a diol 18 in 82\% yield. Two $O$-benzoyl groups were removed under Zemplén's conditions to provide a psicouridine 19 in 94\% yield. ${ }^{21}$

In conclusion, we have demonstrated $\beta$-selective $N$-psicofuranosylation of pyrimidine bases and $S$-psicofuranosylation of thiols. $N$-Glycosidation of 1 with uracil, thymine, and $N^{4}$-benzoylcytosine resulted in 8 7:1 ratio of $\beta$ - and $\alpha$-anomers. The modification of 3,4-O-protecting group to 3-pentylidene yielded increased the $\beta$-selectivities for $S$-glycosidation of psicofuranose $15(\beta: \alpha=7: 1)$. These results will be valuable for the synthesis of other $N$ - and $S$-psicofuranoside derivatives. 


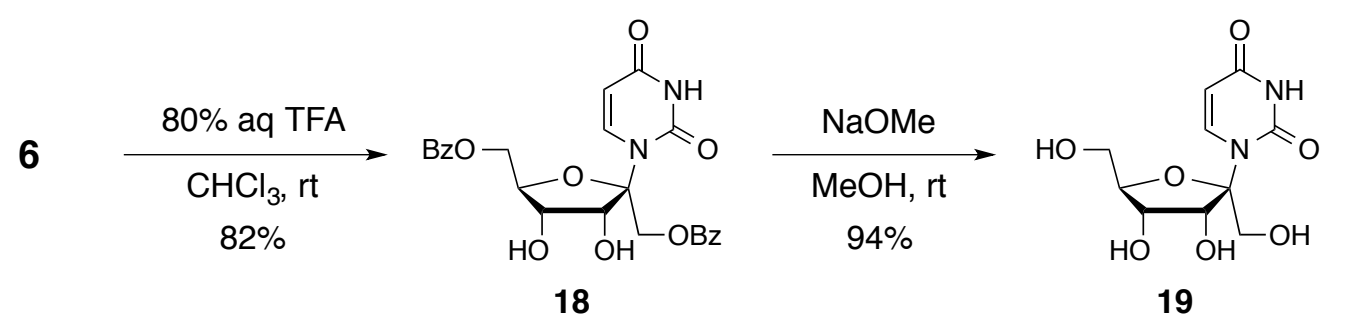

Scheme 5. Deprotection of $\beta$-D-psicofuranoside 6

\section{EXPERIMENTAL}

General information. Specific rotations were measured on a JASCO P-2200 or DIP-370 polarimeter using $\mathrm{CHCl}_{3}$ or $\mathrm{H}_{2} \mathrm{O}$ as a solvent. ${ }^{1} \mathrm{H}$ NMR and ${ }^{13} \mathrm{C}$ NMR spectra were measured on JEOL JNM-AL-300 (300 MHz and $75 \mathrm{MHz}$ ), Varian UNITY INOVA $400 \mathrm{NB}$ (400 MHz) spectrometer, or Varian NMR System 500PS SN (500 MHz and $125 \mathrm{MHz}$ ). Chemical shifts $(\delta)$ are reported in parts per million (ppm) relative to the resonance of tetramethylsilane $(0.00 \mathrm{ppm})$ for ${ }^{1} \mathrm{H}$ NMR spectra, and ppm relative to the resonance of the central peak of $\mathrm{CDCl}_{3}(77.0 \mathrm{ppm})$ or to $\mathrm{MeCN}(1.47 \mathrm{ppm})$ when $\mathrm{D}_{2} \mathrm{O}$ was used, for ${ }^{13} \mathrm{C}$ NMR spectra. IR spectra were recorded on a JASCO FT/IR-410 or Shimadzu IRAffinity-1 FT-IR spectrophotometer. High-resolution mass spectra (HRMS) were obtained on a JEOL JMS 303HF spectrometer using fast atom bombardment (FAB) ionization in the dual focusing sector field mode or on a JEOL JMS-T100TD using electrospray ionization (ESI) or direct analysis in real time (DART) ionization in TOF mode. Silica gel (230-400 mesh) was used for flash column chromatography. Analytical thin-layer chromatography (TLC) was performed on glass pre-coated with silica gel $(0.25 \mathrm{~mm}$ thickness). All moisture sensitive reactions were carried out under an argon atmosphere. THF was dried over sodium/benzophenone ketyl, and $\mathrm{CH}_{2} \mathrm{Cl}_{2}$ was dried over $\mathrm{P}_{2} \mathrm{O}_{5}$, and they were distilled prior to use.

\section{General procedure for $N$-glycosidation:}

A stirred solution of pyrimidine base $(0.300 \mathrm{mmol})$ and $N, O$-bis(trimethylsilyl)acetamide $(0.147 \mathrm{~mL}$, $0.600 \mathrm{mmol})$ in $\mathrm{MeCN}(1.0 \mathrm{~mL})$ was heated at reflux for $1 \mathrm{~h}$. The reaction mixture was cooled to $0{ }^{\circ} \mathrm{C}$ and was added donor 1 or $15(0.200 \mathrm{mmol})$ in $\mathrm{CH}_{2} \mathrm{Cl}_{2} \quad(2.0 \mathrm{~mL})$ and trimethylsilyl trifluoromethanesulfonate (TMSOTf, $36.2 \mu \mathrm{L}, 0.200 \mathrm{mmol}$ for 1 and $72.4 \mu \mathrm{L}, 0.400 \mathrm{mmol}$ for 15). The reaction was stirred for the mentioned time at room temperature and quenched with satd. aq. $\mathrm{NaHCO}_{3}$ solution. The aqueous layer was extracted with $\mathrm{CHCl}_{3}$ and the combined organic layers were washed with water and brine, dried $\left(\mathrm{MgSO}_{4}\right)$, and evaporated. The residue was purified by flash column chromatography on silica gel eluted with EtOAc in $n$-hexane to give the desired $N$-glycoside.

\section{General procedure for $S$-glycosidation:}

The donor 1 or $15(0.200 \mathrm{mmol})$ was azeotropically dried with toluene twice. Then, the above donor was 
dissolved in $\mathrm{CH}_{2} \mathrm{Cl}_{2}(2.0 \mathrm{~mL})$ and molecular sieves (MS) $4 \AA$ (for 15 only, $100 \mathrm{mg}$ ) and thiol $(0.300$ mmol) were added to the solution. To this solution was added TMSOTf ( $36.2 \mu \mathrm{L}, 0.200$ mmol for 1 and $72.4 \mu \mathrm{L}, 0.400 \mathrm{mmol}$ for 15$)$ dropwise at $-40{ }^{\circ} \mathrm{C}$. The reaction mixture was warmed to $-20{ }^{\circ} \mathrm{C}$ and stirred for the mentioned time. After completion of the reaction, the reaction mixture was quenched with $\mathrm{Et}_{3} \mathrm{~N}$ $(0.1$ or $0.2 \mathrm{~mL})$ and warmed to room temperature. After filtration through a Celite pad and concentration under vacuum, the residue was purified by flash column chromatography on silica gel eluted with EtOAc in $n$-hexane to give the $S$-glycoside. Stereochemical assignments of anomeric position were performed by comparison with their structurally related $\beta$-D-psicofuranosides ${ }^{10,11}$ (e.g., coupling constants of $J_{3,4}$ and $J_{4,5}$ values in ${ }^{1} \mathrm{H} \mathrm{NMR}$, chemical shifts of $\mathrm{C} 2$ in ${ }^{13} \mathrm{C} \mathrm{NMR}, R_{\mathrm{f}}$ values, etc.).

\section{6- $O$-Benzoyl-3,4- $O$-isopropylidene-D-allitol and 6- $O$-benzoyl-3,4- $O$-isopropylidene-D-altritol (3): To} a mixed solution of alkene $2^{10}(4.90 \mathrm{~g}, 16.8 \mathrm{mmol})$ in acetone and water $(2: 1,34 \mathrm{~mL})$ were added osmium tetroxide solution (4 wt. \% in $\mathrm{H}_{2} \mathrm{O}, 1.02 \mathrm{~mL}, 0.167 \mathrm{mmol}$ ) and 4-methylmorpholine $N$-oxide (2.95 g, 25.2 $\mathrm{mmol}$ ) at room temperature and the reaction was vigorously stirred for 3 days at room temperature. The reaction mixture was quenched with satd. aq. $\mathrm{Na}_{2} \mathrm{~S}_{2} \mathrm{O}_{3}$ solution $(100 \mathrm{~mL})$ and stirred for $30 \mathrm{~min}$. The reaction mixture was extracted with EtOAc $(4 \times 150 \mathrm{~mL})$ and combined organic layers were washed with $1 \mathrm{M} \mathrm{HCl}$, water, and brine (50 mL each), dried over anhydrous $\mathrm{Na}_{2} \mathrm{SO}_{4}$, and concentrated under reduced pressure. The residue was purified by flash column chromatography on silica gel eluted with 65\% EtOAc in $n$-hexane to give triol $3(4.36 \mathrm{~g}, 80 \%)$ as a $10: 1$ diastereomeric mixture. Colorless oil. $R_{\mathrm{f}}=0.28$ for major isomer and 0.13 for minor isomer $\left(60 \%\right.$ EtOAc in $n$-hexane). ${ }^{1} \mathrm{H}$ NMR $\left(300 \mathrm{MHz}, \mathrm{CDCl}_{3}\right) \delta$ (major): 8.07-8.03 (2H, m), 7.58-7.51 (1H, m), 7.45-7.38 (2H, m), $4.71(1 \mathrm{H}, \mathrm{d}, J=11.9 \mathrm{~Hz}), 4.61(1 \mathrm{H}$, br s), 4.46 (1H, br s), 4.39 (1H, dd, $J=11.9,5.7 \mathrm{~Hz}), 4.23-4.09$ (3H, m), 3.93-3.81 (2H, m), 3.72-3.65 $(1 \mathrm{H}, \mathrm{m}), 3.06(1 \mathrm{H}$, br s), $1.38(3 \mathrm{H}, \mathrm{s}), 1.31(3 \mathrm{H}, \mathrm{s}) ; \delta$ (minor): 8.08-8.04 $(2 \mathrm{H}, \mathrm{m}), 7.61-7.55(1 \mathrm{H}, \mathrm{m})$, $7.48-7.42(2 \mathrm{H}, \mathrm{m}), 4.71(1 \mathrm{H}, \mathrm{dd}, J=11.5,2.1 \mathrm{~Hz}), 4.41(1 \mathrm{H}, \mathrm{dd}, J=11.5,6.2 \mathrm{~Hz}), 4.37-4.30(1 \mathrm{H}, \mathrm{m})$, $4.25(1 \mathrm{H}, \mathrm{dd}, J=6.1,2.6 \mathrm{~Hz}), 4.21-4.14(1 \mathrm{H}, \mathrm{m}), 4.14(1 \mathrm{H}, \mathrm{dd}, J=9.4,6.1 \mathrm{~Hz}), 3.82(1 \mathrm{H}, \mathrm{dd}, J=11.3$, $5.6 \mathrm{~Hz}), 3.76-3.69(1 \mathrm{H}, \mathrm{m}), 3.53(1 \mathrm{H}, \mathrm{br} \mathrm{s}), 2.96(1 \mathrm{H}, \mathrm{d}, J=7.5 \mathrm{~Hz}), 2.39(1 \mathrm{H}, \mathrm{br} \mathrm{s}), 1.50(3 \mathrm{H}, \mathrm{s}), 1.37$ (3H, s). ${ }^{13} \mathrm{C} \mathrm{NMR}\left(75 \mathrm{MHz}, \mathrm{CDCl}_{3}\right) \delta$ (major): 167.3, 133.2, 129.7, 129.7, 128.4, 109.2, 77.3, 76.8, 69.5, 68.5, 67.1, 64.3, 27.9, 25.4. IR (film): 3406, 2987, 1721, $1452 \mathrm{~cm}^{-1}$. HRMS (FAB) $m / z:[\mathrm{M}+\mathrm{Na}]^{+}$calcd for $\mathrm{C}_{16} \mathrm{H}_{22} \mathrm{O}_{7} \mathrm{Na}, 349.1263$; found, 349.1266.

6-O-Benzoyl-3,4- $\boldsymbol{O}$-isopropylidene-D-psicofuranose (4): A mixture of triol $\mathbf{3}$ (4.36 g, $13.4 \mathrm{mmol})$ and di-n-butyltin(IV) oxide $(3.50 \mathrm{~g}, 14.1 \mathrm{mmol})$ in toluene $(168 \mathrm{~mL})$ was heated at reflux overnight with Dean-Stark apparatus attached to the reaction vessel, then cooled to ambient temperature and evaporated. The residue was dissolved in $\mathrm{CHCl}_{3}(168 \mathrm{~mL})$ and was added $N$-bromosuccinimide $(2.51 \mathrm{~g}, 14.1 \mathrm{mmol})$ 
at $0{ }^{\circ} \mathrm{C}$. The reaction was stirred for $1 \mathrm{~h}$ at room temperature and the reaction mixture was quenched with satd. aq. $\mathrm{NaHCO}_{3}$ solution $(100 \mathrm{~mL})$. The aqueous layer was extracted with EtOAc $(3 \times 200 \mathrm{~mL})$ and combined organic layers were washed with water and brine $\left(100 \mathrm{~mL}\right.$ each), dried over anhydrous $\mathrm{Na}_{2} \mathrm{SO}_{4}$ and concentrated in vacuo. The crude product was purified by flash column chromatography on silica gel eluted with $35 \%$ EtOAc in $n$-hexane to give furanose $4(3.40 \mathrm{~g}, 79 \%)$ as a $2: 1$ mixture of $\beta$ - and $\alpha$-anomers. Colorless oil. $R_{\mathrm{f}}=0.44\left(60 \%\right.$ EtOAc in $n$-hexane). ${ }^{1} \mathrm{H}$ NMR $\left(300 \mathrm{MHz}, \mathrm{CDCl}_{3}\right) \delta$ $(\beta \text {-anomer })^{22}: 8.07-8.01(2 \mathrm{H}, \mathrm{m}), 7.60-7.53(1 \mathrm{H}, \mathrm{m}), 7.47-7.41(2 \mathrm{H}, \mathrm{m}), 4.86\left(1 \mathrm{H}, \mathrm{dd}, J_{3,4}=5.9, J_{4,5}=\right.$ $1.3 \mathrm{~Hz}, \mathrm{H}-4), 4.68\left(1 \mathrm{H}, \mathrm{d}, J_{3,4}=5.9 \mathrm{~Hz}, \mathrm{H}-3\right), 4.61\left(1 \mathrm{H}, \mathrm{dd}, J_{6 \mathrm{a}, 6 \mathrm{~b}}=10.8, J_{5,6 \mathrm{a}}=6.8 \mathrm{~Hz}, \mathrm{H}-6 \mathrm{a}\right), 4.56-4.40$ $(1 \mathrm{H}, \mathrm{m}, \mathrm{H}-5), 4.37\left(1 \mathrm{H}, \mathrm{dd}, J_{6 \mathrm{a}, 6 \mathrm{~b}}=10.8, J_{5,6 \mathrm{~b}}=6.4 \mathrm{~Hz}, \mathrm{H}-6 \mathrm{~b}\right), 4.02(1 \mathrm{H}, \mathrm{br}$ s, 2-OH), 3.79 (2H, br s, H-1), $2.44\left(1 \mathrm{H}\right.$, br s, 1-OH), $1.49(3 \mathrm{H}, \mathrm{s}), 1.34(3 \mathrm{H}, \mathrm{s}) ; \delta(\alpha \text {-anomer })^{22}: 8.07-8.01(2 \mathrm{H}, \mathrm{m}), 7.60-7.53(1 \mathrm{H}, \mathrm{m})$, $7.47-7.41(2 \mathrm{H}, \mathrm{m}), 4.82\left(1 \mathrm{H}, \mathrm{d}, J_{3,4}=7.2 \mathrm{~Hz}, \mathrm{H}-3\right), 4.74\left(1 \mathrm{H}, \mathrm{dd}, J_{3,4}=7.2, J_{4,5}=3.5 \mathrm{~Hz}, \mathrm{H}-4\right), 4.56-4.40$ (3H, m, H-5, 6a, 6b), 4.30 (1H, br s, 2-OH), 3.61 (2H, br s, H-1), 2.44 (1H, br s, 1-OH), 1.63 (3H, s), 1.41 $(3 \mathrm{H}, \mathrm{s}) .{ }^{13} \mathrm{C} \mathrm{NMR}\left(75 \mathrm{MHz}, \mathrm{CDCl}_{3}\right) \delta(\beta \text {-anomer })^{22}: 166.6,133.2,129.7,128.4,113.1,106.7,85.3,84.0$,

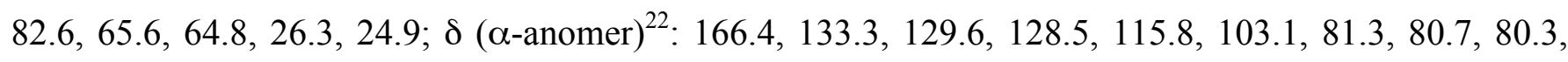
65.4, 64.3, 26.5, 24.9. IR (film): 3443, 2939, 1725, $1602 \mathrm{~cm}^{-1}$. HRMS (FAB) $\mathrm{m} / z$ : $[\mathrm{M}+\mathrm{Na}]^{+}$calcd for $\mathrm{C}_{16} \mathrm{H}_{20} \mathrm{O}_{7} \mathrm{Na}, 347.1107$; found, 347.1104 .

1,6-Di-O-benzoyl-3,4- $\boldsymbol{O}$-isopropylidene-D-psicofuranose (5): To a solution of furanose 4 (735 mg, 2.27 mmol) in $\mathrm{CH}_{2} \mathrm{Cl}_{2}(20 \mathrm{~mL})$ were added triethylamine $(0.947 \mathrm{~mL}, 6.81 \mathrm{mmol})$ and benzoyl chloride $(0.525$ $\mathrm{mL}, 4.54 \mathrm{mmol}$ ) at $0{ }^{\circ} \mathrm{C}$ and the reaction was stirred for $20 \mathrm{~min}$ at the same temperature. The reaction mixture was quenched with $\mathrm{MeOH}(1 \mathrm{~mL})$ and stirred for $10 \mathrm{~min}$ at room temperature, then added satd. aq. $\mathrm{NaHCO}_{3}$ solution $(20 \mathrm{~mL})$. The aqueous layer was extracted with EtOAc $(3 \times 20 \mathrm{~mL})$ and combined organic layers were dried over anhydrous $\mathrm{Na}_{2} \mathrm{SO}_{4}$ and evaporated. The residue was purified by flash column chromatography on silica gel eluted with $15 \%$ EtOAc in $n$-hexane to give furanose 5 (936 mg, 96\%) as a 3:1 mixture of $\beta$ - and $\alpha$-anomers. The spectroscopic data showed the same value as those reported previously. ${ }^{10}$

1-(1,6-O-Benzoyl-3,4- $O$-isopropylidene- $\beta$ - and $\alpha$-D-psicofuranosyl)uracil (6$\beta$ and $6 \alpha)$ : According to the general procedure for the $N$-glycosidation, a mixture of compounds $\mathbf{6} \beta$ and $\mathbf{6} \boldsymbol{\alpha}$ was obtained from $\mathbf{1}$ and uracil in 68\% yield in a 7:1 ratio. White solid. Eluent for column: 50\% EtOAc in $n$-hexane. $R_{\mathrm{f}}=0.37$ (60\% EtOAc in $n$-hexane). ${ }^{1} \mathrm{H}$ NMR $\left(400 \mathrm{MHz}, \mathrm{C}_{6} \mathrm{D}_{6}-\mathrm{CDCl}_{3}, 5: 2\right) \delta$ ( $\beta$-anomer) ${ }^{22}: 9.07$ (1H, br s, NH), 7.93-7.79 (4H, m), 7.31 (1H, d, $\left.J_{5,6}=8.2 \mathrm{~Hz}, \mathrm{H}-6\right), 7.23-7.01(6 \mathrm{H}, \mathrm{m}), 5.38\left(1 \mathrm{H}, \mathrm{d}, J_{3}\right.$ ', $\left.4^{\prime}=6.0 \mathrm{~Hz}, \mathrm{H}-3^{\prime}\right)$, $5.21\left(1 \mathrm{H}, \mathrm{d}, J_{5,6}=8.4 \mathrm{~Hz}, \mathrm{H}-5\right), 5.10\left(1 \mathrm{H}, \mathrm{d}, J_{1}^{\prime} \mathrm{a}, 1^{\prime} \mathrm{b}=12.1 \mathrm{~Hz}, \mathrm{H}-1\right.$ 'a $), 4.86\left(1 \mathrm{H}, \mathrm{d}, J_{1}^{\prime} \mathrm{a}, 1^{\prime} \mathrm{b}=12.1 \mathrm{~Hz}\right.$, $\left.\mathrm{H}-1^{\prime} \mathrm{b}\right), 4.43\left(1 \mathrm{H}\right.$, ddd, $\left.J_{5^{\prime}, 6^{\prime} \mathrm{b}}=3.3, J_{5^{\prime}, 6^{\prime} \mathrm{a}}=2.7, J_{4^{\prime}, 5^{\prime}}=1.5 \mathrm{~Hz}, \mathrm{H}-5^{\prime}\right), 4.40\left(1 \mathrm{H}, \mathrm{dd}, J_{3^{\prime}, 4^{\prime}}=6.0, J_{4^{\prime}, 5^{\prime}}=1.5\right.$ 


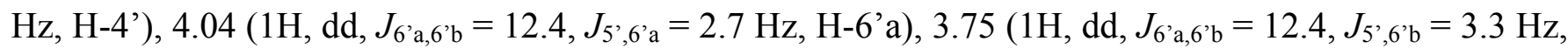
H-6’b), $1.46(3 \mathrm{H}, \mathrm{s}), 1.15(3 \mathrm{H}, \mathrm{s}) ; \delta$ ( $\alpha$-anomer $)^{22}: 9.33(1 \mathrm{H}$, br s, NH), 8.01-7.90 (4H, m), $7.34(1 \mathrm{H}, \mathrm{d}$, $\left.J_{5,6}=8.2 \mathrm{~Hz}, \mathrm{H}-6\right), 7.23-7.01(6 \mathrm{H}, \mathrm{m}), 5.44\left(1 \mathrm{H}, \mathrm{d}, J_{5,6}=8.2 \mathrm{~Hz}, \mathrm{H}-5\right), 4.94\left(1 \mathrm{H}, \mathrm{d}, J_{3^{\prime}, 4^{\prime}}=5.7 \mathrm{~Hz}, \mathrm{H}-3^{\prime}\right)$,

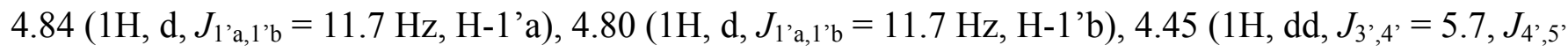
$=4.2 \mathrm{~Hz}, \mathrm{H}-4$ ') , 4.27-4.19 (3H, m, H-5', 6'a, 6’b), $1.18(3 \mathrm{H}, \mathrm{s}), 1.11(3 \mathrm{H}, \mathrm{s}) .{ }^{13} \mathrm{C} \mathrm{NMR}\left(75 \mathrm{MHz}, \mathrm{CDCl}_{3}\right)$ $\delta(\beta \text {-anomer })^{22}: 165.7,165.6,162.6,150.0,140.3,133.9,133.3,129.6,129.3,129.0,128.8,128.7,128.5$, 114.0, 101.1, 99.8, 86.7, 83.8, 81.8, 65.0, 64.6, 25.9, 24.4. IR (KBr): 3448, 1726, $1686 \mathrm{~cm}^{-1}$. HRMS (FAB) $m / z:[\mathrm{M}+\mathrm{Na}]^{+}$calcd for $\mathrm{C}_{27} \mathrm{H}_{26} \mathrm{~N}_{2} \mathrm{O}_{9} \mathrm{Na}, 545.1536$; found, 545.1530.

1-(1,6-O-Benzoyl-3,4- $O$-isopropylidene- $\beta$ - and $\alpha$-D-psicofuranosyl)thymine $(7 \beta$ and $7 \alpha)$ : According to the general procedure for the $N$-glycosidation, a mixture of compounds $7 \boldsymbol{\beta}$ and $7 \boldsymbol{\alpha}$ was obtained from 1 and thymine in 91\% yield in a 8:1 ratio. White solid. Eluent for column: $60 \%$ EtOAc in $n$-hexane. $R_{\mathrm{f}}=$ $0.41\left(60 \%\right.$ EtOAc in $n$-hexane). ${ }^{1} \mathrm{H}$ NMR $\left(300 \mathrm{MHz}, \mathrm{CDCl}_{3}\right) \delta(\beta \text {-anomer })^{22}: 7.87-7.35(11 \mathrm{H}, \mathrm{m}), 5.54$ $\left(1 \mathrm{H}, \mathrm{d}, J_{3^{\prime}, 4^{\prime}}=6.2 \mathrm{~Hz}, \mathrm{H}-3^{\prime}\right), 4.98\left(1 \mathrm{H}, \mathrm{dd}, J_{3^{\prime}, 4^{\prime}}=6.2, J_{4^{\prime}, 5^{\prime}}=1.3 \mathrm{~Hz}, \mathrm{H}-4^{\prime}\right), 4.96\left(1 \mathrm{H}, \mathrm{d}, J_{1}^{\prime}{ }^{\prime}\right.$, $1^{\prime}{ }^{\prime} \mathrm{b}=12.1 \mathrm{~Hz}$, H-1'a), $4.87\left(1 \mathrm{H}, \mathrm{ddd}, J_{5^{\prime}, 6^{\prime} \mathrm{b}}=3.1, J_{5^{\prime}, 6^{\prime} \mathrm{a}}=2.8, J_{4^{\prime}, 5^{\prime}}=1.3 \mathrm{~Hz}, \mathrm{H}-5^{\prime}\right), 4.76\left(1 \mathrm{H}, \mathrm{dd}, J_{6^{\prime} \mathrm{a}, 6^{\prime} \mathrm{b}}=12.6, J_{5^{\prime}, 6^{\prime} \mathrm{a}}=\right.$

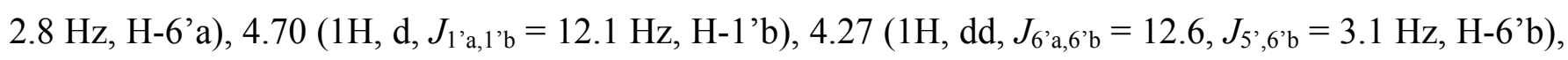
$1.68(3 \mathrm{H}, \mathrm{s}), 1.61(3 \mathrm{H}, \mathrm{s}), 1.45(3 \mathrm{H}, \mathrm{s}) ; \delta(\alpha \text {-anomer })^{22}: 8.29(1 \mathrm{H}$, br s, NH), 8.06-7.90 (4H, m), 7.64$7.50(3 \mathrm{H}, \mathrm{m}), 7.48-7.33$ (4H, m), 5.20 (1H, d, J=5.4 Hz), 5.03-4.83 (3H, m), 4.59-4.44 (3H, m), 1.97 $(3 \mathrm{H}, \mathrm{s}), 1.43(3 \mathrm{H}, \mathrm{s}), 1.38(3 \mathrm{H}, \mathrm{s}) .{ }^{13} \mathrm{C} \mathrm{NMR}\left(75 \mathrm{MHz}, \mathrm{CDCl}_{3}\right) \delta(\beta \text {-anomer })^{22}: 165.5,163.0,149.9,136.3$, 134.0, 133.2, 129.6, 129.5, 129.4, 128.9, 128.7, 128.5, 128.4, 109.3, 99.7, 86.7, 83.9, 81.4, 65.1, 64.4, 25.9, 24.5, 12.0. IR (KBr): 2963, $1686 \mathrm{~cm}^{-1}$. HRMS (FAB) $m / z:[\mathrm{M}+\mathrm{Na}]^{+}$calcd for $\mathrm{C}_{28} \mathrm{H}_{28} \mathrm{~N}_{2} \mathrm{O}_{9} \mathrm{Na}$, 559.1693; found, 559.1686.

\section{$N^{4}$-Benzoyl-1-(1,6-O-benzoyl-3,4- $O$-isopropylidene- $\beta$ - and $\alpha$-D-psicofuranosyl)cytosine (8$\beta$ and}

$\mathbf{8} \boldsymbol{\alpha})$ : According to the general procedure for the $N$-glycosidation, a mixture of compounds $\mathbf{8} \boldsymbol{\beta}$ and $\mathbf{8} \boldsymbol{\alpha}$ was obtained from 1 and $N^{4}$-benzoylcytosine in 72\% yield in a 7:1 ratio. White solid. Eluent for column: $60 \%$ EtOAc in $n$-hexane. $R_{\mathrm{f}}=0.18\left(60 \%\right.$ EtOAc in $n$-hexane). ${ }^{1} \mathrm{H}$ NMR $\left(300 \mathrm{MHz}, \mathrm{CDCl}_{3}\right) \delta(\beta \text {-anomer })^{22}$ : $8.75\left(1 \mathrm{H}\right.$, br s, NH), $8.09\left(1 \mathrm{H}, \mathrm{d}, J_{5,6}=7.7 \mathrm{~Hz}, \mathrm{H}-6\right), 7.97-7.28(16 \mathrm{H}, \mathrm{m}), 5.56\left(1 \mathrm{H}, \mathrm{d}, J_{3^{\prime}, 4^{\prime}}=6.1 \mathrm{~Hz}\right.$, H-3'), $5.03\left(1 \mathrm{H}, \mathrm{d}, J_{1^{\prime} \mathrm{a}, 1^{\prime} \mathrm{b}}=11.9 \mathrm{~Hz}, \mathrm{H}-1^{\prime} \mathrm{a}\right), 4.96\left(1 \mathrm{H}, \mathrm{dd}, J_{3^{\prime}, 4^{\prime}}=6.1, J_{4^{\prime}, 5^{\prime}}=1.0 \mathrm{~Hz}, \mathrm{H}-4^{\prime}\right), 4.90(1 \mathrm{H}, \mathrm{ddd}$, $\left.J_{5^{\prime}, 6^{\prime} \mathrm{b}}=2.9, J_{5}{ }^{\prime}, 6^{\prime} \mathrm{a}=2.4, J_{4^{\prime}, 5^{\prime}}=1.0 \mathrm{~Hz}, \mathrm{H}-5^{\prime}\right), 4.90\left(1 \mathrm{H}, \mathrm{d}, J_{1}{ }^{\prime} \mathrm{a}, 1^{\prime} \mathrm{b}=11.9 \mathrm{~Hz}, \mathrm{H}-1^{\prime} \mathrm{b}\right), 4.67\left(1 \mathrm{H}, \mathrm{dd}, J_{6^{\prime} \mathrm{a}, 6^{\prime} \mathrm{b}}=\right.$ $12.7, J_{5^{\prime}, 6^{\prime} \mathrm{a}}=2.4 \mathrm{~Hz}, \mathrm{H}-6$ 'a $), 4.33\left(1 \mathrm{H}, \mathrm{dd}, J_{6^{\prime} \mathrm{a}, 6^{\prime} \mathrm{b}}=12.7, J_{5}{ }^{\prime}, 6^{\prime} \mathrm{b}=2.9 \mathrm{~Hz}, \mathrm{H}-6\right.$ 'b $), 1.70(3 \mathrm{H}, \mathrm{s}), 1.45(3 \mathrm{H}$, s); $\delta(\alpha \text {-anomer })^{22}: 8.69\left(1 \mathrm{H}\right.$, br s, NH), $8.16\left(1 \mathrm{H}, \mathrm{d}, J_{5,6}=7.6 \mathrm{~Hz}, \mathrm{H}-6\right), 7.94-7.10(16 \mathrm{H}, \mathrm{m}), 5.29(1 \mathrm{H}, \mathrm{d}$, $J=5.6 \mathrm{~Hz}), 5.06-4.78(3 \mathrm{H}, \mathrm{m}), 4.54-4.37(3 \mathrm{H}, \mathrm{m}), 1.27(3 \mathrm{H}, \mathrm{s}), 1.26(3 \mathrm{H}, \mathrm{s}) .{ }^{13} \mathrm{C} \mathrm{NMR}(75 \mathrm{MHz}$, $\left.\mathrm{CDCl}_{3}\right) \delta(\beta \text {-anomer })^{22}: 165.6,165.4,162.2,145.1,133.3,133.1,133.0,129.5,129.4,129.1,128.9,128.4$, 
128.4, 127.5, 113.8, 100.4, 86.4, 83.9, 81.7, 64.8, 64.6, 25.9, 24.4. IR (KBr): $3423,1720 \mathrm{~cm}^{-1}$. HRMS (ESI) $m / z:[\mathrm{M}+\mathrm{Na}]^{+}$calcd for $\mathrm{C}_{34} \mathrm{H}_{31} \mathrm{~N}_{3} \mathrm{O}_{9} \mathrm{Na}, 648.1958$; found, 648.1978.

\section{Phenyl 1,6- $O$-benzoyl-3,4- $O$-isopropylidene-2-thio- $\beta$ - and $\alpha$-D-psicofuranoside $(9 \beta$ and $9 \alpha)$ :} According to the general procedure for the $S$-glycosidation, a mixture of compounds $9 \beta$ and $9 \alpha$ was obtained from 1 and thiophenol in 79\% yield in a 3:1 ratio. Colorless oil. Eluent for column: 15\% EtOAc in $n$-hexane. $R_{\mathrm{f}}=0.33$ for $9 \beta$ and 0.29 for $9 \alpha\left(20 \%\right.$ EtOAc in $n$-hexane). ${ }^{1} \mathrm{H}$ NMR $\left(400 \mathrm{MHz}, \mathrm{CDCl}_{3}\right) \delta$ $(\beta \text {-anomer })^{22}: 8.14-8.10$ (4H, m), 7.62-7.54 (4H, m), 7.49-7.45 (4H, m), 7.31-7.27 (1H, m), 7.24-7.19 $(2 \mathrm{H}, \mathrm{m}), 4.98\left(1 \mathrm{H}, \mathrm{dd}, J_{3,4}=5.9, J_{4,5}=2.6 \mathrm{~Hz}, \mathrm{H}-4\right), 4.85\left(1 \mathrm{H}, \mathrm{d}, J_{3,4}=5.9 \mathrm{~Hz}, \mathrm{H}-3\right), 4.79\left(1 \mathrm{H}, \mathrm{dd}, J_{6 \mathrm{a}, 6 \mathrm{~b}}=\right.$ $\left.11.4, J_{5,6 \mathrm{a}}=6.6 \mathrm{~Hz}, \mathrm{H}-6 \mathrm{a}\right), 4.77\left(1 \mathrm{H}, \mathrm{dd}, J_{6 \mathrm{a}, 6 \mathrm{~b}}=11.4, J_{5,6 \mathrm{~b}}=7.0 \mathrm{~Hz}, \mathrm{H}-6 \mathrm{~b}\right), 4.66\left(1 \mathrm{H}, \mathrm{ddd}, J_{5,6 \mathrm{~b}}=7.0, J_{5,6 \mathrm{a}}\right.$ $\left.=6.6, J_{4,5}=2.6 \mathrm{~Hz}, \mathrm{H}-5\right), 4.52\left(1 \mathrm{H}, \mathrm{d}, J_{1 \mathrm{a}, 1 \mathrm{~b}}=11.5 \mathrm{~Hz}, \mathrm{H}-1 \mathrm{a}\right), 4.27\left(1 \mathrm{H}, \mathrm{d}, J_{1 \mathrm{a}, 1 \mathrm{~b}}=11.5 \mathrm{~Hz}, \mathrm{H}-1 \mathrm{~b}\right), 1.50$ $(3 \mathrm{H}, \mathrm{s}), 1.35(3 \mathrm{H}, \mathrm{s}) ; \delta(\alpha \text {-anomer })^{22}: 8.05-8.03(2 \mathrm{H}, \mathrm{m}), 8.00-7.97(2 \mathrm{H}, \mathrm{m}), 7.61-7.53(4 \mathrm{H}, \mathrm{m}), 7.42-$ $7.32(5 \mathrm{H}, \mathrm{m}), 7.30-7.26(2 \mathrm{H}, \mathrm{m}), 5.07\left(1 \mathrm{H}, \mathrm{d}, J_{3,4}=7.7 \mathrm{~Hz}, \mathrm{H}-3\right), 4.89\left(1 \mathrm{H}, \mathrm{ddd}, J_{5,6 \mathrm{~b}}=6.0, J_{4,5}=5.9\right.$, $\left.J_{5,6 \mathrm{a}}=3.3 \mathrm{~Hz}, \mathrm{H}-5\right), 4.78\left(1 \mathrm{H}, \mathrm{dd}, J_{3,4}=7.7, J_{4,5}=5.9 \mathrm{~Hz}, \mathrm{H}-4\right), 4.68\left(1 \mathrm{H}, \mathrm{dd}, J_{6 \mathrm{a}, 6 \mathrm{~b}}=12.1, J_{5,6 \mathrm{a}}=3.3 \mathrm{~Hz}\right.$, H-6a), $4.56\left(1 \mathrm{H}, \mathrm{dd}, J_{6 \mathrm{a}, 6 \mathrm{~b}}=12.1, J_{5,6 \mathrm{~b}}=6.0 \mathrm{~Hz}, \mathrm{H}-6 \mathrm{~b}\right), 4.48\left(1 \mathrm{H}, \mathrm{d}, J_{1 \mathrm{a}, 1 \mathrm{~b}}=11.9 \mathrm{~Hz}, \mathrm{H}-1 \mathrm{a}\right), 4.24(1 \mathrm{H}, \mathrm{d}$, $\left.J_{1 \mathrm{a}, 1 \mathrm{~b}}=11.9 \mathrm{~Hz}, \mathrm{H}-1 \mathrm{~b}\right), 1.73(3 \mathrm{H}, \mathrm{s}), 1.41(3 \mathrm{H}, \mathrm{s}) .{ }^{13} \mathrm{C} \mathrm{NMR}\left(125 \mathrm{MHz}, \mathrm{CDCl}_{3}\right) \delta(\beta \text {-anomer })^{22}: 166.2$, 165.7, 135.5 (2C), 133.2, 133.0, 129.82 (2C), 129.80 (3C), 129.7, 129.6, 129.1, 128.9 (2C), 128.42 (2C), 128.37 (2C), 114.1, 97.6, 86.2, 85.0, 83.0, 64.8, 62.8, 26.5, 25.3; $\delta$ ( $\alpha$-anomer) ${ }^{22}: 166.1,165.8,136.3(2 \mathrm{C})$, 133.2 (2C), 130.3, 130.0, 129.8, 129.7, 129.59 (2C), 129.57, 128.9, 128.7 (2C), 128.45 (2C), 128.42, 128.37, 117.7, 95.8, 83.6, 81.2, 79.9, 66.1, 63.7, 25.9, 25.4. IR (film): 3063, 2990, 2940, $1721 \mathrm{~cm}^{-1}$. HRMS (DART) $m / z$ : [M+NH$]^{+}$calcd for $\mathrm{C}_{29} \mathrm{H}_{32} \mathrm{NO}_{7} \mathrm{~S}, 538.1900$; found, 538.1921.

\section{1-Dodecyl 1,6-O-benzoyl-3,4- $O$-isopropylidene-2-thio- $\beta$ - and $\alpha$-D-psicofuranoside (10 $\beta$ and $10 \alpha)$ :}

According to the general procedure for the $S$-glycosidation, compounds $\mathbf{1 0} \beta$ and $\mathbf{1 0} \boldsymbol{\alpha}$ were obtained from 1 and 1-dodecanethiol in 56\% and 27\% yields, respectively. Eluent for column: $7 \%(\mathbf{1 0 \beta})$ and 10\% (10 $\alpha)$ EtOAc in $n$-hexane. $R_{\mathrm{f}}=0.52$ for $\mathbf{1 0} \beta$ and 0.45 for $\mathbf{1 0} \alpha$ (20\% EtOAc in $n$-hexane). 10 $\beta$ : Colorless oil. $[\alpha]^{17}{ }_{\mathrm{D}}-31.9\left(\mathrm{c} 1.00, \mathrm{CHCl}_{3}\right) .{ }^{1} \mathrm{H}$ NMR $\left(500 \mathrm{MHz}, \mathrm{CDCl}_{3}\right)$ 8: 8.14-8.04 (4H, m), 7.63-7.53 (2H, m), $7.51-7.41(4 \mathrm{H}, \mathrm{m}), 4.96\left(1 \mathrm{H}, \mathrm{dd}, J_{3,4}=5.9, J_{4,5}=2.2 \mathrm{~Hz}, \mathrm{H}-4\right), 4.74\left(1 \mathrm{H}, \mathrm{d}, J_{1 \mathrm{a}, 1 \mathrm{~b}}=11.4 \mathrm{~Hz}, \mathrm{H}-1 \mathrm{a}\right), 4.68$ $\left(1 \mathrm{H}, \mathrm{dd}, J_{6 \mathrm{a}, 6 \mathrm{~b}}=10.7, J_{5,6 \mathrm{a}}=6.3 \mathrm{~Hz}, \mathrm{H}-6 \mathrm{a}\right), 4.66\left(1 \mathrm{H}, \mathrm{d}, J_{3,4}=5.9 \mathrm{~Hz}, \mathrm{H}-3\right), 4.62\left(1 \mathrm{H}, \mathrm{dd}, J_{6 \mathrm{a}, 6 \mathrm{~b}}=10.7\right.$, $\left.J_{5,6 \mathrm{~b}}=7.1 \mathrm{~Hz}, \mathrm{H}-6 \mathrm{~b}\right), 4.59\left(1 \mathrm{H}, \mathrm{ddd}, J_{5,6 \mathrm{~b}}=7.1, J_{5,6 \mathrm{a}}=6.3, J_{4,5}=2.2 \mathrm{~Hz}, \mathrm{H}-5\right), 4.53\left(1 \mathrm{H}, \mathrm{d}, J_{1 \mathrm{a}, 1 \mathrm{~b}}=11.4\right.$ Hz, H-1a), $2.73(1 \mathrm{H}, \mathrm{dt}, J=11.8,7.5 \mathrm{~Hz}, \mathrm{SCHH}), 2.64(1 \mathrm{H}, \mathrm{dt}, J=11.8,7.6 \mathrm{~Hz}, \mathrm{SCH} H), 1.53(3 \mathrm{H}, \mathrm{s}$, $\left.\mathrm{OCCH}_{3}\right), 1.52-1.45\left(2 \mathrm{H}, \mathrm{m}, \mathrm{SCH}_{2} \mathrm{CH}_{2}\right), 1.37\left(3 \mathrm{H}, \mathrm{s}, \mathrm{OCCH}_{3}\right), 1.30-1.12(18 \mathrm{H}, \mathrm{m}), 0.88(3 \mathrm{H}, \mathrm{t}, J=7.0$ $\left.\mathrm{Hz}, \mathrm{CH}_{2} \mathrm{CH}_{3}\right) .{ }^{13} \mathrm{C} \mathrm{NMR}\left(125 \mathrm{MHz}, \mathrm{CDCl}_{3}\right) \delta: 166.1,165.9,133.2,133.0,130.1,129.83$ (2C), 129.75 
(2C), 129.69, 128.4 (2C), 128.3 (2C), 113.9, 95.0, 86.5, 85.1, 83.3, 65.0, 63.2, 31.9, 29.61, 29.59, 29.50, 29.4, 29.3, 29.23, 29.20, 29.1, 28.0, 26.7, 25.4, 22.7, 14.1. IR (film): 2926, 2855, $1724 \mathrm{~cm}^{-1}$. HRMS (ESI) $m / z:[\mathrm{M}+\mathrm{Na}]^{+}$calcd for $\mathrm{C}_{35} \mathrm{H}_{48} \mathrm{O}_{7} \mathrm{SNa}, 635.3018$; found, 635.2991. 10 $\alpha$ : Colorless oil. $R_{\mathrm{f}}=0.45(20 \%$ EtOAc in $n$-hexane). $[\alpha]^{17} \mathrm{D}+53.3\left(c 0.80, \mathrm{CHCl}_{3}\right) .{ }^{1} \mathrm{H}$ NMR $\left(500 \mathrm{MHz}, \mathrm{CDCl}_{3}\right) \delta: 8.09-7.89(4 \mathrm{H}, \mathrm{m})$, $7.62-7.48(2 \mathrm{H}, \mathrm{m}), 7.43-7.29(4 \mathrm{H}, \mathrm{m}), 5.01\left(1 \mathrm{H}, \mathrm{d}, J_{3,4}=7.6 \mathrm{~Hz}, \mathrm{H}-3\right), 4.76\left(1 \mathrm{H}, \mathrm{dd}, J_{3,4}=7.6, J_{4,5}=5.1\right.$ $\mathrm{Hz}, \mathrm{H}-4), 4.67\left(1 \mathrm{H}, \mathrm{d}, J_{1 \mathrm{a}, 1 \mathrm{~b}}=12.1 \mathrm{~Hz}, \mathrm{H}-1 \mathrm{a}\right), 4.72-4.44(3 \mathrm{H}, \mathrm{m}, \mathrm{H}-1 \mathrm{~b}, 5,6 \mathrm{a}), 4.51\left(1 \mathrm{H}, \mathrm{dd}, J_{6 \mathrm{a}, 6 \mathrm{~b}}=12.9\right.$, $\left.J_{5,6 \mathrm{~b}}=5.9 \mathrm{~Hz}, \mathrm{H}-6 \mathrm{~b}\right), 2.78-2.58\left(2 \mathrm{H}, \mathrm{m}, \mathrm{SCH} \mathrm{S}_{2}\right), 1.68\left(3 \mathrm{H}, \mathrm{s}, \mathrm{OCCH}_{3}\right), 1.65-1.58\left(2 \mathrm{H}, \mathrm{m}, \mathrm{SCH}_{2} \mathrm{CH}_{2}\right), 1.38$ $\left(3 \mathrm{H}, \mathrm{s}, \mathrm{OCCH}_{3}\right), 1.44-1.21(18 \mathrm{H}, \mathrm{m}), 0.88\left(3 \mathrm{H}, \mathrm{t}, J=6.8 \mathrm{~Hz}, \mathrm{CH}_{2} \mathrm{CH}_{3}\right) .{ }^{13} \mathrm{C} \mathrm{NMR}\left(125 \mathrm{MHz}, \mathrm{CDCl}_{3}\right) \delta$ : 166.2, 165.9, 133.13, 133.07, 129.7, 129.62 (2C), 129.61 (2C), 129.5, 128.44 (2C), 128.38 (2C), 117.5, 94.2, 83.4, 81.1, 79.9, 66.2, 63.6, 31.9, 30.0, 29.64, 29.61, 29.58, 29.52, 29.3, 29.20, 29.17, 27.3, 25.7, 25.4, 22.7, 14.1. IR (film): 2926, 2855, $1724 \mathrm{~cm}^{-1}$. HRMS (ESI) $\mathrm{m} / z:[\mathrm{M}+\mathrm{Na}]^{+}$calcd for $\mathrm{C}_{35} \mathrm{H}_{48} \mathrm{O}_{7} \mathrm{SNa}$, 635.3018; found, 635.3013 .

1,6-Di-O-(tert-butyldiphenylsilyl)-D-psicofuranose (11): To a solution of D-psicose (1.00 g, $5.56 \mathrm{mmol})$ in pyridine $(30 \mathrm{~mL})$ were added tert-butyldiphenylchlorosilane (TBDPSCl; $4.34 \mathrm{~mL}, 16.7 \mathrm{mmol})$ and 4-(dimethylamino)pyridine (DMAP; $339 \mathrm{mg}, 2.78 \mathrm{mmol}$ ) at room temperature and the reaction mixture was stirred at the same temperature for $24 \mathrm{~h}$. The reaction mixture was quenched with $1 \mathrm{M} \mathrm{HCl}$, extracted with EtOAc twice. Combined organic extracts were washed with water, satd. aq. $\mathrm{NaHCO}_{3}$ solution, and brine. After drying over anhydrous $\mathrm{MgSO}_{4}$ and evaporation of solvent, the residue was purified by flash column chromatography on silica gel eluted with 30\% EtOAc in $n$-hexane to give silyl ether $11(3.41 \mathrm{~g}$, 93\%). White amorphous solid. $R_{\mathrm{f}}=0.36$ (40\% EtOAc in $n$-hexane). ${ }^{1} \mathrm{H}$ NMR (500 MHz, $\left.\mathrm{CDCl}_{3}\right) \delta: 7.71-$ $7.67(2 \mathrm{H}, \mathrm{m}), 7.67-7.63(2 \mathrm{H}, \mathrm{m}), 7.63-7.59$ (4H, m), 7.43-7.37 (4H, m), 7.37-7.28 (8H, m), 4.22 (1H, br $\mathrm{s}, 2-\mathrm{OH}), 4.25-4.16(2 \mathrm{H}, \mathrm{m}, \mathrm{H}-4,5), 4.03\left(1 \mathrm{H}, \mathrm{dd}, J_{3,3-\mathrm{OH}}=7.6, J_{3,4}=5.6 \mathrm{~Hz}, \mathrm{H}-3\right), 3.76\left(1 \mathrm{H}, \mathrm{dd}, J_{6 \mathrm{a}, 6 \mathrm{~b}}=\right.$ 11.0, $\left.J_{5,6 \mathrm{a}}=3.5 \mathrm{~Hz}, \mathrm{H}-6 \mathrm{a}\right), 3.76(2 \mathrm{H}, \mathrm{s}, \mathrm{H}-1), 3.69\left(1 \mathrm{H}, \mathrm{dd}, J_{6 \mathrm{a}, 6 \mathrm{~b}}=11.0, J_{5,6 \mathrm{~b}}=4.7 \mathrm{~Hz}, \mathrm{H}-6 \mathrm{~b}\right), 3.07(1 \mathrm{H}, \mathrm{d}$, $\left.J_{3,3-\mathrm{OH}}=7.6 \mathrm{~Hz}, 3-\mathrm{OH}\right), 2.84\left(1 \mathrm{H}, \mathrm{d}, J_{4,4-\mathrm{OH}}=6.6 \mathrm{~Hz}, 4-\mathrm{OH}\right), 1.06(9 \mathrm{H}, \mathrm{s}), 0.99(9 \mathrm{H}, \mathrm{s}) .{ }^{13} \mathrm{C} \mathrm{NMR}(125$ $\mathrm{MHz}, \mathrm{CDCl}_{3}$ ) $\delta: 135.54$ (2C), 135.47 (6C), 132.9, 132.8, 132.6, 132.5, 129.87, 129.85, 129.81, 129.7, 127.79 (2C), 127.77 (2C), 127.75 (2C), 127.72 (2C), 102.8, 84.0, 72.5, 72.4, 66.7, 64.1, 26.8 (3C), 26.7 (3C), 19.2, 19.1. IR (KBr): 3449, 2932, $2869 \mathrm{~cm}^{-1}$. HRMS (ESI) $m / z:[\mathrm{M}+\mathrm{Na}]^{+}$calcd for $\mathrm{C}_{38} \mathrm{H}_{48} \mathrm{O}_{6} \mathrm{Si}_{2} \mathrm{Na}$, 679.2887; found, 679.2895 .

1,6-Di-O-benzoyl-3,4-O-(3-pentylidene)-D-psicofuranose (14): To triol 11 (2.40 g, $3.65 \mathrm{mmol})$ and trimethyl orthoformate $(1.20 \mathrm{~mL}, 11.0 \mathrm{mmol})$ in 3-pentanone $(12 \mathrm{~mL})$ was added $p$-toluenesulfonic acid monohydrate $\left(p-\mathrm{TsOH} \cdot \mathrm{H}_{2} \mathrm{O} ; 69.4 \mathrm{mg}, 0.365 \mathrm{mmol}\right)$ at room temperature and the reaction mixture was stirred at room temperature for $1 \mathrm{~h}$. The reaction mixture was quenched by adding triethylamine $(0.2 \mathrm{~mL})$ 
and concentrated under vacuum. The residue was purified by flash column chromatography on silica gel eluted with 5\% EtOAc in $n$-hexane to afford desired ketal $12\left(R_{\mathrm{f}}=0.54\right.$ and 0.46 with $15 \%$ EtOAc in $n$-hexane) contaminated with its methyl glycoside $20\left(R_{\mathrm{f}}=0.54\right.$ with $15 \%$ EtOAc in $n$-hexane). Fractions eluted with 7\% EtOAc in $n$-hexane gave 2,3-O-(3-pentylidene) byproduct $21^{20}(\sim 5 \%)$. The above product 12 was dissolved in THF $(37 \mathrm{~mL})$ and treated with tetrabutylammonium fluoride solution (1 $\mathrm{M}$ in THF, $14.6 \mathrm{~mL}, 14.6 \mathrm{mmol}$ ) at room temperature. The reaction mixture was stirred at room temperature for $1.5 \mathrm{~h}$ and quenched with $\mathrm{CaCO}_{3}(3.0 \mathrm{~g})$, Dowex 50W×8-400 (9.0 g), and $\mathrm{MeOH}^{23}(21 \mathrm{~mL})$ and further stirred at room temperature for $1 \mathrm{~h}$. The resultant suspension was filtered through a Celite pad and concentrated under vacuum to give residue, which was passed through a short plug of silica gel eluted first with $\mathrm{CHCl}_{3}$ (discarded) and then with $10 \% \mathrm{MeOH}$ in EtOAc (collected). Removal of solvent provided a crude product 13 (contaminated with its methyl glycoside 22), which was used for the next step without further purification. To a stirred solution of the above triol 13, pyridine $(8.86 \mathrm{~mL}, 110 \mathrm{mmol})$, and DMAP (224 $\mathrm{mg}, 1.83 \mathrm{mmol})$ in $\mathrm{CH}_{2} \mathrm{Cl}_{2}(37 \mathrm{~mL})$ was added benzoyl chloride $(4.24 \mathrm{~mL}, 36.5 \mathrm{mmol})$ dropwise at $0{ }^{\circ} \mathrm{C}$, and the resultant mixture was stirred at $0{ }^{\circ} \mathrm{C}$ for $30 \mathrm{~min}$. The reaction mixture was quenched by adding $\mathrm{MeOH}(8 \mathrm{~mL})$ at $0{ }^{\circ} \mathrm{C}$ and stirred for $10 \mathrm{~min}$ at room temperature prior to the addition of water $(10 \mathrm{~mL})$. After removal of solvent, the residue was dissolved in EtOAc and $1 \mathrm{M} \mathrm{HCl}$, and the aqueous layer was extracted with EtOAc twice. Combined organics were washed with water, satd. aq. $\mathrm{NaHCO}_{3}$ solution, and brine. After drying over anhydrous $\mathrm{MgSO}_{4}$ and concentration under vacuum, the residue was purified by flash column chromatography on silica gel (10\% then $15 \%$ EtOAc in $n$-hexane) to afford benzoate 14 (1.15 g, $69 \%$ in 3 steps, $\alpha: \beta=7: 1)$ as a colorless oil. The methyl glycoside byproduct $\mathbf{2 3}^{20}(\sim 15 \%)$ was removed at this stage. 14: $R_{\mathrm{f}}=0.25\left(20 \%\right.$ EtOAc in $n$-hexane). ${ }^{1} \mathrm{H}$ NMR $\left(500 \mathrm{MHz}, \mathrm{CDCl}_{3}\right) \delta$ $(\beta \text {-anomer })^{22}: 8.10-8.04(4 \mathrm{H}, \mathrm{m}), 7.59-7.51(2 \mathrm{H}, \mathrm{m}), 7.46-7.37(4 \mathrm{H}, \mathrm{m}), 4.87\left(1 \mathrm{H}, \mathrm{dd}, J_{3,4}=5.9, J_{4,5}=\right.$ $1.5 \mathrm{~Hz}, \mathrm{H}-4), 4.79\left(1 \mathrm{H}, \mathrm{dd}, J_{6 \mathrm{a}, 6 \mathrm{~b}}=11.4, J_{5,6 \mathrm{a}}=8.4 \mathrm{~Hz}, \mathrm{H}-6 \mathrm{a}\right), 4.73\left(1 \mathrm{H}, \mathrm{d}, J_{3,4}=5.9 \mathrm{~Hz}, \mathrm{H}-3\right), 4.62(1 \mathrm{H}, \mathrm{d}$, $\left.J_{1 \mathrm{a}, 1 \mathrm{~b}}=11.6 \mathrm{~Hz}, \mathrm{H}-1 \mathrm{a}\right), 4.56\left(1 \mathrm{H}, \mathrm{d}, J_{1 \mathrm{a}, 1 \mathrm{~b}}=11.6 \mathrm{~Hz}, \mathrm{H}-1 \mathrm{~b}\right), 4.54\left(1 \mathrm{H}, \mathrm{ddd}, J_{5,6 \mathrm{a}}=8.4, J_{5,6 \mathrm{~b}}=6.0, J_{4,5}=1.5\right.$ Hz, H-5), $4.32\left(1 \mathrm{H}, \mathrm{dd}, J_{6 \mathrm{a}, 6 \mathrm{~b}}=11.4, J_{5,6 \mathrm{~b}}=6.0 \mathrm{~Hz}, \mathrm{H}-6 \mathrm{~b}\right), 4.20(1 \mathrm{H}, \mathrm{s}, \mathrm{OH}), 1.80-1.66(2 \mathrm{H}, \mathrm{m}), 1.62(2 \mathrm{H}$, q, $J=7.5 \mathrm{~Hz}), 0.95(3 \mathrm{H}, \mathrm{t}, J=7.5 \mathrm{~Hz}), 0.89(3 \mathrm{H}, \mathrm{t}, J=7.5 \mathrm{~Hz}) ; \delta(\alpha \text {-anomer })^{22}: 8.04-8.00(4 \mathrm{H}, \mathrm{m}), 7.59-$ $7.51(2 \mathrm{H}, \mathrm{m}), 7.46-7.37(4 \mathrm{H}, \mathrm{m}), 4.83-4.75(2 \mathrm{H}, \mathrm{m}), 4.56-4.45(4 \mathrm{H}, \mathrm{m}), 4.45\left(1 \mathrm{H}, \mathrm{d}, J_{1 \mathrm{a}, 1 \mathrm{~b}}=11.6 \mathrm{~Hz}\right.$, $\mathrm{H}-1 \mathrm{a}), 4.42\left(1 \mathrm{H}, \mathrm{d}, J_{1 \mathrm{a}, 1 \mathrm{~b}}=11.6 \mathrm{~Hz}, \mathrm{H}-1 \mathrm{~b}\right), 1.91-1.85(2 \mathrm{H}, \mathrm{m}), 1.71-1.66(2 \mathrm{H}, \mathrm{m}), 1.04(3 \mathrm{H}, \mathrm{t}, J=7.5$ $\mathrm{Hz}), 0.89(3 \mathrm{H}, \mathrm{t}, J=7.5 \mathrm{~Hz}) .{ }^{13} \mathrm{C} \mathrm{NMR}\left(125 \mathrm{MHz} \mathrm{CDCl}_{3}\right) \delta(\beta \text {-anomer })^{22}: 166.8,166.5,133.2,133.1$, 129.77 (2C), 129.76 (2C), 129.68, 129.63, 128.32 (2C), 128.26 (2C), 117.7, 106.1, 85.5, 85.0, 82.5, 65.92, 65.86, 29.4, 29.0, 8.3, 7.6; $\delta$ ( $\alpha$-anomer) ${ }^{22}:$ 166.2, 165.9, 133.6-128.4 (12C), 120.8, 101.5, 81.2, 81.1, 79.9, 66.3, 64.1, 28.9, 28.7, 8.4, 7.9. IR (film): 3437, 2974, 2943, $1701 \mathrm{~cm}^{-1}$. HRMS (DART) $m / z$ : [M+H $\left.-\mathrm{H}_{2} \mathrm{O}\right]^{+}$calcd for $\mathrm{C}_{25} \mathrm{H}_{27} \mathrm{O}_{7}, 439.1757$; found, 439.1764 . 
Benzyl [1,6-di-O-benzoyl-3,4-O-(3-pentylidene)-D-psicofuranosyl] phthalate (15): To a solution of alcohol 14 (235 mg, $0.515 \mathrm{mmol})$ and benzyl hydrogen phthalate $(396 \mathrm{mg}, 1.55 \mathrm{mmol})$ in $\mathrm{CH}_{2} \mathrm{Cl}_{2}(5 \mathrm{~mL})$ were added $N, N^{\prime}$-dicyclohexylcarbodiimide (319 mg, $\left.1.55 \mathrm{mmol}\right)$ and DMAP (62.8 $\left.\mathrm{mg}, 0.515 \mathrm{mmol}\right)$ at $0{ }^{\circ} \mathrm{C}$, and the reaction mixture was stirred at room temperature for $24 \mathrm{~h}$. The resultant suspension was diluted with $\mathrm{CH}_{2} \mathrm{Cl}_{2}$ and passed through a Celite pad $\left(\mathrm{CH}_{2} \mathrm{Cl}_{2}\right)$. Combined organics were washed with $5 \%$ $\mathrm{Na}_{2} \mathrm{CO}_{3}$ aq and brine, dried over anhydrous $\mathrm{MgSO}_{4}$, and concentrated in vacuo. The residue was purified by flash column chromatography on silica gel eluted with $20 \%$ EtOAc in $n$-hexane to give the title compound 15 (330 mg, 92\%, mostly $\beta$-anomer). Colorless syrup. $R_{\mathrm{f}}=0.63\left(10 \% \mathrm{Et}_{2} \mathrm{O}\right.$ in $\left.\mathrm{CHCl}_{3}\right)$. ${ }^{1} \mathrm{H}$ NMR (500 MHz, $\left.\mathrm{CDCl}_{3}\right)$ ઈ: 8.09-8.03 (2H, m), 8.03-7.97 (2H, m), 7.83-7.76 (1H, m), 7.64-7.57 (1H, m), 7.56-7.49 (2H, m), 7.49-7.43 (2H, m), 7.42-7.27 (9H, m), $5.39\left(1 \mathrm{H}, \mathrm{d}, J_{3,4}=6.0 \mathrm{~Hz}, \mathrm{H}-3\right), 5.31(1 \mathrm{H}$, $\mathrm{d}, J=12.3 \mathrm{~Hz}, \mathrm{CHHPh}), 5.26(1 \mathrm{H}, \mathrm{d}, J=12.3 \mathrm{~Hz}, \mathrm{CH} H \mathrm{Ph}), 5.22\left(1 \mathrm{H}, \mathrm{d}, J_{1 \mathrm{a}, 1 \mathrm{~b}}=12.0 \mathrm{~Hz}, \mathrm{H}-1 \mathrm{a}\right), 4.99(1 \mathrm{H}$, $\left.\mathrm{d}, J_{1 \mathrm{a}, 1 \mathrm{~b}}=12.0 \mathrm{~Hz}, \mathrm{H}-1 \mathrm{~b}\right), 4.96\left(1 \mathrm{H}, \mathrm{dd}, J_{3,4}=6.0, J_{4,5}=1.9 \mathrm{~Hz}, \mathrm{H}-4\right), 4.69\left(1 \mathrm{H}, \mathrm{ddd}, J_{5,6 \mathrm{a}}=7.0, J_{5,6 \mathrm{~b}}=\right.$ $\left.6.6, J_{4,5}=1.9 \mathrm{~Hz}, \mathrm{H}-5\right), 4.42\left(1 \mathrm{H}, \mathrm{dd}, J_{6 \mathrm{a}, 6 \mathrm{~b}}=11.5, J_{5,6 \mathrm{a}}=7.0 \mathrm{~Hz}, \mathrm{H}-6 \mathrm{a}\right), 4.37\left(1 \mathrm{H}, \mathrm{dd}, J_{6 \mathrm{a}, 6 \mathrm{~b}}=11.5, J_{5,6 \mathrm{~b}}=\right.$ $6.6 \mathrm{~Hz}, \mathrm{H}-6 \mathrm{~b}), 1.88-1.72(2 \mathrm{H}, \mathrm{m}), 1.64(2 \mathrm{H}, \mathrm{q}, J=7.5 \mathrm{~Hz}), 0.99(3 \mathrm{H}, \mathrm{t}, J=7.5 \mathrm{~Hz}), 0.91(3 \mathrm{H}, \mathrm{t}, J=7.5$

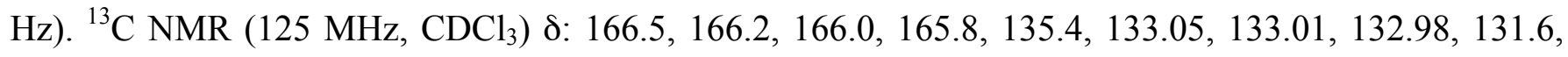
130.9, 130.4, 129.9, 129.8 (2C), 129.7 (2C), 129.6, 129.4, 128.53, 128.51 (2C), 128.3 (5C), 128.20 (2C), 118.2, 112.5, 86.3, 84.9, 82.3, 67.4, 64.6, 63.2, 29.1, 28.9, 8.4, 7.8. IR (KBr): 2974, 2941, 1736, $1719 \mathrm{~cm}^{-1}$. HRMS (ESI) $m / z$ : [M+Na] $]^{+}$calcd for $\mathrm{C}_{40} \mathrm{H}_{38} \mathrm{O}_{11} \mathrm{Na}, 717.2312$; found, 717.2318.

Phenyl 1,6- $O$-benzoyl-3,4- $O$-(3-pentylidene)-2-thio- $\beta$ - and $\alpha$-D-psicofuranoside (16 $\beta$ and $16 \alpha)$ : According to the general procedure for the $S$-glycosidation, a mixture of compounds $16 \beta$ and $16 \alpha$ was obtained from 15 and thiophenol in 83\% yield in a 7:1 ratio. Colorless oil. Eluent for column: 8\% EtOAc in $n$-hexane. $R_{\mathrm{f}}=0.38$ for $\mathbf{1 6} \beta$ and 0.36 for $\mathbf{1 6} \boldsymbol{\alpha}\left(20 \%\right.$ EtOAc in $n$-hexane). ${ }^{1} \mathrm{H}$ NMR (500 MHz, $\left.\mathrm{CDCl}_{3}\right)$ $\delta(\beta \text {-anomer })^{22}: 8.16-8.08(4 \mathrm{H}, \mathrm{m}), 7.62-7.54(4 \mathrm{H}, \mathrm{m}), 7.50-7.44(4 \mathrm{H}, \mathrm{m}), 7.30-7.26(1 \mathrm{H}, \mathrm{m}), 7.23-7.17$ $(2 \mathrm{H}, \mathrm{m}), 4.99\left(1 \mathrm{H}, \mathrm{dd}, J_{3,4}=6.0, J_{4,5}=2.6 \mathrm{~Hz}, \mathrm{H}-4\right), 4.85\left(1 \mathrm{H}, \mathrm{d}, J_{3,4}=6.0 \mathrm{~Hz}, \mathrm{H}-3\right), 4.84\left(1 \mathrm{H}, \mathrm{dd}, J_{6 \mathrm{a}, 6 \mathrm{~b}}\right.$ $\left.=11.4, J_{5,6 \mathrm{a}}=6.7 \mathrm{~Hz}, \mathrm{H}-6 \mathrm{a}\right), 4.81\left(1 \mathrm{H}, \mathrm{dd}, J_{6 \mathrm{a}, 6 \mathrm{~b}}=11.4, J_{5,6 \mathrm{~b}}=7.3 \mathrm{~Hz}, \mathrm{H}-6 \mathrm{~b}\right), 4.68\left(1 \mathrm{H}, \mathrm{ddd}, J_{5,6 \mathrm{~b}}=7.3\right.$, $\left.J_{5,6 \mathrm{a}}=6.7, J_{4,5}=2.6 \mathrm{~Hz}, \mathrm{H}-5\right), 4.58\left(1 \mathrm{H}, \mathrm{d}, J_{1 \mathrm{a}, 1 \mathrm{~b}}=11.3 \mathrm{~Hz}, \mathrm{H}-1 \mathrm{a}\right), 4.27\left(1 \mathrm{H}, \mathrm{d}, J_{1 \mathrm{a}, 1 \mathrm{~b}}=11.3 \mathrm{~Hz}, \mathrm{H}-1 \mathrm{~b}\right)$, $1.81-1.69(2 \mathrm{H}, \mathrm{m}), 1.61(2 \mathrm{H}, \mathrm{q}, J=7.5 \mathrm{~Hz}), 0.93(3 \mathrm{H}, \mathrm{t}, J=7.4 \mathrm{~Hz}), 0.85(3 \mathrm{H}, \mathrm{t}, J=7.5 \mathrm{~Hz}) ; \delta$ $(\alpha \text {-anomer })^{22}$ : 8.07-8.04 (2H, m), 8.00-7.97 (2H, m), 7.63-7.18 $(11 \mathrm{H}, \mathrm{m}), 5.05\left(1 \mathrm{H}, \mathrm{d}, J_{3,4}=7.6 \mathrm{~Hz}\right.$, $\mathrm{H}-3), 4.83-4.66(3 \mathrm{H}, \mathrm{m}, \mathrm{H}-4,5,6 \mathrm{a}), 4.55\left(1 \mathrm{H}, \mathrm{dd}, J_{6 \mathrm{a}, 6 \mathrm{~b}}=12.1, J_{5,6 \mathrm{~b}}=6.4 \mathrm{~Hz}, \mathrm{H}-6 \mathrm{~b}\right), 4.48\left(1 \mathrm{H}, \mathrm{d}, J_{1 \mathrm{a}, 1 \mathrm{~b}}=\right.$ $12.0 \mathrm{~Hz}, \mathrm{H}-1 \mathrm{a}), 4.21\left(1 \mathrm{H}, \mathrm{d}, J_{1 \mathrm{a}, 1 \mathrm{~b}}=12.0 \mathrm{~Hz}, \mathrm{H}-1 \mathrm{~b}\right), 1.70-1.58(4 \mathrm{H}, \mathrm{m}), 0.95-0.83(6 \mathrm{H}, \mathrm{m}) .{ }^{13} \mathrm{C} \mathrm{NMR}$ $\left(125 \mathrm{MHz}, \mathrm{CDCl}_{3}\right) \delta(\beta \text {-anomer })^{22}: 166.2,165.6,135.4(2 \mathrm{C}), 133.2,133.0,130.0,129.81(2 \mathrm{C}), 129.79$ (2C), 129.69, 129.61, 129.0, 128.9 (2C), 128.41 (2C), 128.37 (2C), 118.4, 98.0, 86.4, 85.6, 83.1, 65.1, $62.7,29.2,29.1,8.3,7.8 ; \delta(\alpha \text {-anomer })^{22}: 166.2,165.7,136.3(2 \mathrm{C}), 133.2,133.1,130.4,130.0-128.3$ 
(13C), 122.1, 95.9, 83.9, 81.3, 79.6, 66.4, 63.8, 29.0, 28.9, 8.3, 7.8. IR (film): 2974, $1734 \mathrm{~cm}^{-1}$. HRMS (ESI) $m / z:[\mathrm{M}+\mathrm{K}]^{+}$calcd for $\mathrm{C}_{31} \mathrm{H}_{32} \mathrm{O}_{7} \mathrm{SK}, 587.1506$; found, 587.1477.

\section{1-Dodecyl 1,6-O-benzoyl-3,4-O-(3-pentylidene)-2-thio- $\beta$ - and $\alpha$-D-psicofuranoside (17 $\beta$ and $17 \alpha)$ :}

According to the general procedure for the $S$-glycosidation, compounds $17 \beta$ and $17 \alpha$ were obtained from 15 and 1-dodecanethiol in $63 \%$ and 9\% yields, respectively. Eluent for column: $4 \%(\mathbf{1 7} \beta)$ and $5 \%(\mathbf{1 7} \boldsymbol{\alpha})$ EtOAc in $n$-hexane. $R_{\mathrm{f}}=0.57$ for $\mathbf{1 7} \beta$ and 0.49 for $\mathbf{1 7} \boldsymbol{\alpha}(20 \%$ EtOAc in $n$-hexane). $\mathbf{1 7} \beta$ : Colorless syrup. $[\alpha]^{21}{ }_{\mathrm{D}}-21.5\left(c\right.$ 1.02, $\left.\mathrm{CHCl}_{3}\right) .{ }^{1} \mathrm{H}$ NMR $\left(500 \mathrm{MHz}, \mathrm{CDCl}_{3}\right) \delta: 8.14-8.05(4 \mathrm{H}, \mathrm{m}), 7.62-7.53(2 \mathrm{H}, \mathrm{m})$, $7.50-7.42(4 \mathrm{H}, \mathrm{m}), 4.95\left(1 \mathrm{H}, \mathrm{dd}, J_{3,4}=6.0, J_{4,5}=2.2 \mathrm{~Hz}, \mathrm{H}-4\right), 4.76\left(1 \mathrm{H}, \mathrm{d}, J_{1 \mathrm{a}, 1 \mathrm{~b}}=11.3 \mathrm{~Hz}, \mathrm{H}-1 \mathrm{a}\right), 4.68$ $\left(1 \mathrm{H}, \mathrm{dd}, J_{6 \mathrm{a}, 6 \mathrm{~b}}=10.9, J_{5,6 \mathrm{a}}=6.7 \mathrm{~Hz}, \mathrm{H}-6 \mathrm{a}\right), 4.65\left(1 \mathrm{H}, \mathrm{dd}, J_{6 \mathrm{a}, 6 \mathrm{~b}}=10.9, J_{5,6 \mathrm{~b}}=7.0 \mathrm{~Hz}, \mathrm{H}-6 \mathrm{~b}\right), 4.65(1 \mathrm{H}, \mathrm{d}$, $\left.J_{3,4}=6.0 \mathrm{~Hz}, \mathrm{H}-3\right), 4.61\left(1 \mathrm{H}, \mathrm{ddd}, J_{5,6 \mathrm{~b}}=7.0, J_{5,6 \mathrm{a}}=6.7, J_{4,5}=2.2 \mathrm{~Hz}, \mathrm{H}-5\right), 4.55\left(1 \mathrm{H}, \mathrm{d}, J_{1 \mathrm{a}, 1 \mathrm{~b}}=11.3 \mathrm{~Hz}\right.$, $\mathrm{H}-1 \mathrm{~b}), 2.73(1 \mathrm{H}, \mathrm{dt}, J=11.7,7.6 \mathrm{~Hz}), 2.64(1 \mathrm{H}, \mathrm{dt}, J=11.7,7.6 \mathrm{~Hz}), 1.84-1.69(2 \mathrm{H}, \mathrm{m}), 1.67-1.60(2 \mathrm{H}$, $\mathrm{m}), 1.53-1.43(2 \mathrm{H}, \mathrm{m}), 1.33-1.09(18 \mathrm{H}, \mathrm{m}), 0.96(3 \mathrm{H}, \mathrm{t}, J=7.4 \mathrm{~Hz}), 0.88(3 \mathrm{H} \times 2, \mathrm{t}, J=7.2 \mathrm{~Hz}) .{ }^{13} \mathrm{C}$ NMR (125 MHz, $\mathrm{CDCl}_{3}$ ) $\delta: 166.2,165.9,133.2,133.0,130.1,129.80$ (2C), 129.76 (2C), 129.73, 128.38 (2C), 128.32 (2C), 118.1, 95.3, 86.6, 85.5, 83.4, 65.3, 63.3, 31.9, 29.61, 29.59, 29.49, 29.45, 29.32, 29.30, 29.22 (2C), 29.19, 29.12, 27.9, 22.7, 14.1, 8.3, 7.8. IR (film): 2926, 1724, $1452 \mathrm{~cm}^{-1}$. HRMS (ESI) $\mathrm{m} / z$ : $[\mathrm{M}+\mathrm{Na}]^{+}$calcd for $\mathrm{C}_{37} \mathrm{H}_{52} \mathrm{O}_{7} \mathrm{SNa}, 663.3331$; found, 663.3328. 17 $\alpha$ : Colorless syrup. ${ }^{1} \mathrm{H}$ NMR (500 MHz,

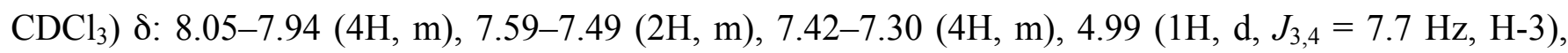
$4.72\left(1 \mathrm{H}, \mathrm{dd}, J_{3,4}=7.7, J_{4,5}=6.0 \mathrm{~Hz}, \mathrm{H}-4\right), 4.66\left(1 \mathrm{H}, \mathrm{d}, J_{1 \mathrm{a}, 1 \mathrm{~b}}=12.0 \mathrm{~Hz}, \mathrm{H}-1 \mathrm{a}\right), 4.64-4.57(2 \mathrm{H}, \mathrm{m}, \mathrm{H}-1 \mathrm{~b}$, 6a), 4.57-4.53 (1H, m, H-5), $4.51\left(1 \mathrm{H}, \mathrm{dd}, J_{6 \mathrm{a}, 6 \mathrm{~b}}=11.4, J_{5,6 \mathrm{~b}}=5.2 \mathrm{~Hz}, \mathrm{H}-6 \mathrm{~b}\right), 2.72-2.60(2 \mathrm{H}, \mathrm{m}), 1.95$ $(2 \mathrm{H}, \mathrm{q}, J=7.5 \mathrm{~Hz}), 1.70-1.58(4 \mathrm{H}, \mathrm{m}), 1.42-1.35(2 \mathrm{H}, \mathrm{m}), 1.32-1.23(16 \mathrm{H}, \mathrm{m}), 1.05(3 \mathrm{H}, \mathrm{t}, J=7.5 \mathrm{~Hz})$, $0.88(3 \mathrm{H}, \mathrm{t}, J=7.7 \mathrm{~Hz}), 0.88(3 \mathrm{H}, \mathrm{t}, J=6.6 \mathrm{~Hz})$. HRMS (ESI) $m / z:[\mathrm{M}+\mathrm{Na}]^{+}$calcd for $\mathrm{C}_{37} \mathrm{H}_{52} \mathrm{O}_{7} \mathrm{SNa}$, 663.3331; found, 663.3358 .

1-(1,6-O-Benzoyl- $\beta$ - and $\boldsymbol{\alpha}$-D-psicofuranosyl)uracil (18 $\beta$ and $18 \boldsymbol{\alpha})$ : A mixed solution of 6 (659 $\mathrm{mg}$, $1.26 \mathrm{mmol})$ in $\mathrm{CHCl}_{3}(8 \mathrm{~mL})$ and $80 \%$ aq. trifluoroacetic acid $(4.5 \mathrm{~mL})$ was stirred overnight at room temperature. Satd. aq. $\mathrm{NaHCO}_{3}$ was added to the reaction mixture, which was extracted with $\mathrm{CHCl}_{3}$. The whole organic layer was washed with satd. aq. $\mathrm{NaHCO}_{3}$ solution, water, and brine, dried over $\mathrm{MgSO}_{4}$, and concentrated under vacuum. The residue was purified by flash column chromatography on silica gel eluted with $70 \%$ (for $\mathbf{1 8} \boldsymbol{\beta}$ ) to $90 \%$ (for $\mathbf{1 8} \boldsymbol{\alpha}$ ) EtOAc in $n$-hexane to give $\mathbf{1 8} \boldsymbol{\beta}$ (497 $\mathrm{mg}, 82 \%$ ) along with $\mathbf{1 8} \boldsymbol{\alpha} . R_{\mathrm{f}}=0.37$ for $\mathbf{1 8} \beta$ and 0.23 for $\mathbf{1 8} \boldsymbol{\alpha}\left(80 \%\right.$ EtOAc in $n$-hexane). $\mathbf{1 8} \beta$ : White solid. Mp $82-83{ }^{\circ} \mathrm{C}$.

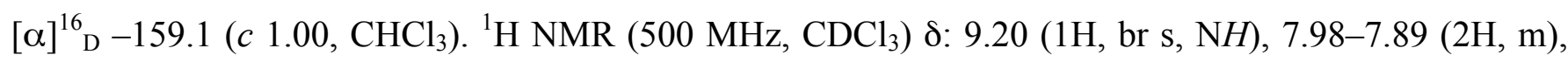
7.89-7.82 (2H, m), $7.73\left(1 \mathrm{H}, \mathrm{d}, J_{5,6}=8.3 \mathrm{~Hz}, \mathrm{H}-6\right), 7.60-7.52(2 \mathrm{H}, \mathrm{m}), 7.47-7.36(4 \mathrm{H}, \mathrm{m}), 5.56(1 \mathrm{H}, \mathrm{d}$, $\left.J_{5,6}=8.3 \mathrm{~Hz}, \mathrm{H}-5\right), 4.97\left(1 \mathrm{H}, \mathrm{d}, J_{3^{\prime}, 4^{\prime}}=3.0 \mathrm{~Hz}, \mathrm{H}-3^{\prime}\right), 4.87\left(1 \mathrm{H}, \mathrm{d}, J_{1^{\prime} \mathrm{a}, 1^{\prime} \mathrm{b}}=12.3 \mathrm{~Hz}, \mathrm{H}-1^{\prime}\right.$ 'a), 4.81 (1H, dd, 
$\left.J_{4^{\prime}, \mathrm{OH}}=5.2, J_{3^{\prime}, 4^{\prime}}=3.0 \mathrm{~Hz}, \mathrm{H}-4^{\prime}\right), 4.77\left(1 \mathrm{H}, \mathrm{d}, J_{1^{\prime} \mathrm{a}, 1^{\prime} \mathrm{b}}=12.3 \mathrm{~Hz}, \mathrm{H}-1^{\prime} \mathrm{b}\right), 4.74\left(1 \mathrm{H}, \mathrm{dd}, J_{6^{\prime} \mathrm{a}, 6^{\prime} \mathrm{b}}=11.6, J_{5^{\prime}, 6^{\prime} \mathrm{a}}\right.$ $=3.5 \mathrm{~Hz}, \mathrm{H}-6$ 'a), 4.73-4.71 (1H, m, H-5'), 4.55 (1H, d, $\left.J_{4}^{\prime}, \mathrm{OH}=5.2 \mathrm{~Hz}, 4^{\prime}-\mathrm{OH}\right), 4.36$ (dd, $J_{6}{ }^{\prime} \mathrm{a}, 6^{\prime} \mathrm{b}=11.6$,

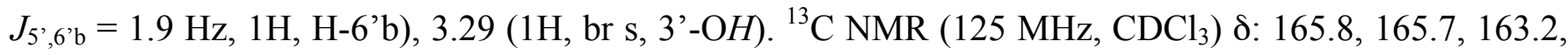
151.7, 141.0, 133.7, 133.4, 129.6 (2C), 129.2 (2C), 129.1, 129.0, 128.6 (2C), 128.5 (2C), 101.7, 98.4, 84.5, 77.7, 73.4, 64.6, 63.7. IR (KBr): 3437 (br), 2951, 2922, 1717, $1684 \mathrm{~cm}^{-1}$. HRMS (ESI) $\mathrm{m} / z$ : $[\mathrm{M}+\mathrm{Na}]^{+}$calcd for $\mathrm{C}_{24} \mathrm{H}_{22} \mathrm{~N}_{2} \mathrm{O}_{9} \mathrm{Na}, 505.1223$; found, 505.1226. 18 $\alpha$ : ${ }^{1} \mathrm{H} \mathrm{NMR}\left(500 \mathrm{MHz}, \mathrm{CDCl}_{3}\right) \delta: 9.25$ (1H, br s, NH), 7.99-7.94 (2H, m), 7.92-7.88 (2H, m), $7.82\left(1 \mathrm{H}, \mathrm{d}, J_{5,6}=8.3 \mathrm{~Hz}, \mathrm{H}-6\right), 7.59-7.49$ (2H, m), 7.43-7.38 (2H, m), 7.36-7.31 (2H, m), $5.81\left(1 \mathrm{H}, \mathrm{d}, J_{5,6}=8.3 \mathrm{~Hz}, \mathrm{H}-5\right), 4.87-4.82(1 \mathrm{H}, \mathrm{m}), 4.80(1 \mathrm{H}$, dd,

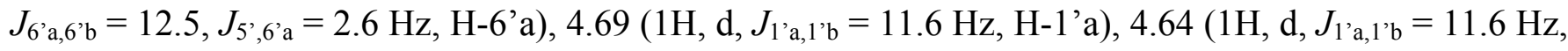
$\left.\mathrm{H}-1{ }^{\prime} \mathrm{b}\right), 4.46\left(1 \mathrm{H}, \mathrm{dd}, J_{6^{\prime} \mathrm{a}, 6^{\prime} \mathrm{b}}=12.5, J_{5^{\prime}, 6^{\prime} \mathrm{b}}=4.9 \mathrm{~Hz}, \mathrm{H}-6\right.$ 'b $), 4.42-4.32(2 \mathrm{H}, \mathrm{m}), 4.25\left(1 \mathrm{H}, \mathrm{ddd}, J_{4^{\prime}, 5^{\prime}}=9.1\right.$, $\left.J_{5^{\prime}, 6^{\prime} \mathrm{b}}=4.8, J_{5^{\prime}, 6^{\prime} \mathrm{a}}=2.6 \mathrm{~Hz}, \mathrm{H}-5^{\prime}\right), 3.15(1 \mathrm{H}, \mathrm{d}, J=9.7 \mathrm{~Hz}, \mathrm{OH})$. HRMS (ESI) $m / z:[\mathrm{M}+\mathrm{Na}]^{+}$calcd for $\mathrm{C}_{24} \mathrm{H}_{22} \mathrm{~N}_{2} \mathrm{O}_{9} \mathrm{Na}, 505.1223$; found, 505.1230.

1- $\boldsymbol{\beta}$-D-Psicofuranosyluracil (19): To a stirred solution of $\mathbf{1 8} \boldsymbol{\beta}(51.5 \mathrm{mg}, 0.107 \mathrm{mmol})$ in $\mathrm{MeOH}(2 \mathrm{~mL})$ was added $\mathrm{NaOMe}(5 \mathrm{M}$ in $\mathrm{MeOH}, 21.4 \mu \mathrm{L}, 0.107 \mathrm{mmol})$ at room temperature and the reaction was stirred for $1 \mathrm{~h}$ at the same temperature. The crude product was purified by Amberlite FPC3500 column $\left(\mathrm{H}_{2} \mathrm{O}\right)$ and lyophilized to give $19(27.6 \mathrm{mg})$ in $94 \%$ yield. White solid. $R_{\mathrm{f}}=0.28\left(30 \% \mathrm{MeOH}\right.$ in $\left.\mathrm{CHCl}_{3}\right)$. Mp 96-99 ${ }^{\circ} \mathrm{C} .[\alpha]^{26}+2.7\left(c 1.00, \mathrm{H}_{2} \mathrm{O}\right) .{ }^{1} \mathrm{H}$ NMR (500 MHz, $\left.\mathrm{CD}_{3} \mathrm{OD}\right) \delta: 8.11\left(1 \mathrm{H}, \mathrm{d}, J_{5,6}=8.3 \mathrm{~Hz}, \mathrm{H}-6\right)$, $5.61\left(1 \mathrm{H}, \mathrm{d}, J_{5,6}=8.3 \mathrm{~Hz}, \mathrm{H}-5\right), 4.73\left(1 \mathrm{H}, \mathrm{d}, J_{3^{\prime}, 4^{\prime}}=5.0 \mathrm{~Hz}, \mathrm{H}-3^{\prime}\right), 4.59(1 \mathrm{H}, \mathrm{br} \mathrm{s}, \mathrm{N} H), 4.20\left(1 \mathrm{H}, \mathrm{d}, J^{\prime}{ }^{\prime} \mathrm{a}, 1^{\prime} \mathrm{b}\right.$ $=12.2 \mathrm{~Hz}, \mathrm{H}-1$ ' 'a), $4.14\left(1 \mathrm{H}, \mathrm{ddd}, J_{4^{\prime}, 5^{\prime}}=6.1, J_{5^{\prime}, 6^{\prime} \mathrm{b}}=4.4, J_{5^{\prime}, 6^{\prime} \mathrm{a}}=3.1 \mathrm{~Hz}, \mathrm{H}-5^{\prime}\right), 4.09$ (1H, dd, $J_{4^{\prime}, 5^{\prime}}$ = 6.1, $\left.J_{3^{\prime}, 4^{\prime}}=5.0 \mathrm{~Hz}, \mathrm{H}-4^{\prime}\right), 3.93\left(1 \mathrm{H}, \mathrm{d}, J_{1}{ }^{\prime} \mathrm{a}, 1^{\prime} \mathrm{b}=12.2 \mathrm{~Hz}, \mathrm{H}-1\right.$ 'b $), 3.81\left(1 \mathrm{H}, \mathrm{dd}, J_{6}{ }^{\prime} \mathrm{a}, 6^{\prime} \mathrm{b}=12.3, J_{5^{\prime}, 6^{\prime} \mathrm{a}}=3.1 \mathrm{~Hz}\right.$, H-6'a), 3.65 (1H, dd, $J_{6}{ }^{\prime}, 6^{\prime}{ }^{\prime} b=12.4, J_{5^{\prime}, 6^{\prime} \mathrm{b}}=4.4 \mathrm{~Hz}, \mathrm{H}-6$ 'b). ${ }^{13} \mathrm{C}$ NMR (125 MHz, $\left.\mathrm{D}_{2} \mathrm{O}\right) \delta: 167.4,152.0$, 143.9, 100.9, 99.9, 84.8, 76.0, 70.6, 62.4, 61.1. IR (KBr): 3449, 1686, $1655 \mathrm{~cm}^{-1}$. HRMS (ESI) $m / z$ : $[\mathrm{M}+\mathrm{Na}]^{+}$calcd for $\mathrm{C}_{10} \mathrm{H}_{14} \mathrm{~N}_{2} \mathrm{O}_{7} \mathrm{Na}, 297.0699$; found, 297.0684 .

\section{ACKNOWLEDGEMENTS}

This work was supported in part by Special Coordination Funds for Promoting Science and Technology from the Japan Science and Technology Agency.

\section{REFERENCES AND NOTES}

1. T. B. Granström, G. Takata, M. Tokuda, and K. Izumori, J. Biosci. Bioeng., 2004, 97, 89.

2. Y. Sun, S. Hayakawa, M. Ogawa, and K. Izumori, Food Control, 2007, 18, 220.

3. T. Matsuo and K. Izumori, Tech. Bull. Fac. Agr. Kagawa Univ., 2006, 58, 27.

4. A. Hossain, F. Yamaguchi, T. Matsuo, I. Tsukamoto, Y. Toyoda, M. Ogawa, Y. Nagata, and M. 
Tokuda, Pharmacol. Ther., 2015, 155, 49.

5. (a) M. Baráth, C.-H. Lin, I. Tvaroška, and J. Hirsch, Chemical Papers, 2015, 69, 348; (b) M. Bella, S. Yan, S. Šesták, S. Kozmon, C.-H. Lin, J. Mucha, and M. Koóš, Eur. J. Org. Chem., 2017, 6179.

6. W. Schroeder and H. Hoeksema, J. Am. Chem. Soc., 1959, 81, 1767.

7. K. Izumori, A. R. Khan, H. Okaya, and T. Tsumura, Biosci., Biotechnol., Biochem., 1993, 57, 1037.

8. (a) H. Itoh, T. Sato, and K. Izumori, J. Ferment. Bioeng., 1995, 80, 101; (b) K. Takeshita, A. Suga, G. Takada, and K. Izumori, J. Biosci. Bioeng., 2000, 90, 453.

9. (a) T. Matsuo, H. Suzuki, M. Hashiguchi, and K. Izumori, J. Nutr. Sci. Vitaminol., 2002, 48, 77; (b) N. Hayashi, T. Yamada, S. Takamine, T. Iida, K. Okuma, and M. Tokuda, J. Funct. Foods, 2014, 11, 152.

10. J. Uenishi and A. Ueda, Tetrahedron: Asymmetry, 2008, 19, 2210.

11. (a) A. Ueda, T. Yamashita, and J. Uenishi, Carbohydr. Res., 2010, 345, 1722; (b) A. Ueda, T. Yamashita, and J. Uenishi, Heterocycles, 2010, 81, 1711; (c) S. Kamitori, A. Ueda, Y. Tahara, H. Yoshida, T. Ishii, and J. Uenishi, Carbohydr. Res., 2011, 346, 1182; (d) J. Uenishi and A. Ueda, Heterocycles, 2009, 77, 1297.

12. Yamanoi et al. has studied stereochemistry of $O$-psicofuranosylation. (a) T. Yamanoi, T. Ishiyama, Y. Oda, S. Matsuda, and M. Watanabe, Heterocycles, 2010, 81, 1141; (b) T. Yamanoi, Y. Oda, T. Ishiyama, and M. Watanabe, Heterocycles, 2016, 93, 55.

13. X. Kong and B. Grindley, J. Carbohydr. Chem., 1993, 12, 557.

14. H. Vorbrüggen and K. Krolikiewicz, Angew. Chem., Int. Ed. Engl., 1975, 14, 421.

15. Chattopadhyaya et al. reported the synthesis of oxetane-nucleosides via $N$-psicofuranosidation of nucleobases with varied anomeric selectivities. M. Bogucka, P. Naus, W. Pathmasiri, J. Barman, and J. Chattopadhyaya, Org. Biomol. Chem., 2005, 3, 4362.

16. Electronic character would also affect the selectivity, because $O$-psicofuranosidation of 1 with 1-pentadecanol gave the corresponding $\beta$-isomer exclusively. ${ }^{20}$

17. Protection of 2,3-diol using 3-pentylidene group in ribose; see, S. Hirano, S. Ichikawa, and A. Matsuda, J. Org. Chem., 2007, 72, 9936.

18. K. S. Kim, Y. J. Lee, H. Y. Kim, S. S. Kang, and S. Y. Kwon, Org. Biomol. Chem., 2004, 2408.

19. The reaction in the absence of MS $4 \AA$ also gave the same $\beta / \alpha$ ratio of $7: 1$ with decreased yield.

20. For details, see the Supporting Information.

21. G. Zemplén and E. Pacsu, Ber. Dtsch. Chem. Ges., 1929, 62, 1613.

22. Assignment was performed from the spectrum of anomeric mixture.

23. Y. Kaburagi and Y. Kishi, Org. Lett., 2007, 9, 723. 